\title{
circPARD3 drives malignant progression and chemoresistance of laryngeal squamous cell carcinoma by inhibiting autophagy through the PRKCl-Akt-mTOR pathway
}

Wei Gao ${ }^{1,2,3,4,5+}$, Huina Guo ${ }^{1,2+}$, Min Niu ${ }^{1,2}$, Xiwang Zheng ${ }^{1,2}$, Yuliang Zhang ${ }^{1,2}$, Xuting Xue ${ }^{1,2}$, Yunfeng Bo ${ }^{6}$, Xiaoya Guan ${ }^{1,2}$, Zhongxun Li ${ }^{1,2}$, Yujia Guo ${ }^{1,2}$, Long He ${ }^{1,2}$, Yu Zhang ${ }^{4,7}$, Li Li ${ }^{5}$, Jimin Cao ${ }^{4,7}$ and Yongyan Wu $\mathrm{Wu}^{1,2,3,4,8^{*}}$ (D)

\begin{abstract}
Background: Laryngeal squamous cell carcinoma (LSCC) is the second most common malignant tumor in head and neck. Autophagy and circular RNAs (circRNAs) play critical roles in cancer progression and chemoresistance. However, the function and mechanism of circRNA in autophagy regulation of LSCC remain unclear.

Methods: The autophagy-suppressive circRNA circPARD3 was identified via RNA sequencing of 107 LSCC tissues and paired adjacent normal mucosal (ANM) tissues and high-content screening. RT-PCR, Sanger sequencing, GPCR and fluorescence in situ hybridization were performed to detect circPARD3 expression and subcellular localization. Biological functions of circPARD3 were assessed by proliferation, migration, invasion, autophagic flux, and chemoresistance assays using in vitro and in vivo models. The mechanism of circPARD3 was investigated by RNA immunoprecipitation, RNA pulldown, luciferase reporter assays, western blotting and immunohistochemical staining.

Results: Autophagy was inhibited in LSCC, and circPARD3 was upregulated in the LSCC tissues $(n=100, p<0.001)$. High circPARD3 level was associated with advanced T stages $(p<0.05)$, N stages $(p=0.001)$, clinical stages $(p<0.001)$, poor differentiation degree $(p=0.025)$, and poor prognosis ( $p=0.002)$ of LSCC patients $(n=100)$. Functionally, cirCPARD3 inhibited autophagy and promoted LSCC cell proliferation, migration, invasion and chemoresistance. We further revealed that activation of the PRKCI-Akt-mTOR pathway through sponging miR-145-5p was the main mechanism of cirCPARD3 inhibited autophagy, promoting LSCC progression and chemoresistance.

(Continued on next page)
\end{abstract}

\footnotetext{
* Correspondence: wuyongyan@sxent.org

${ }^{\dagger}$ Wei Gao and Huina Guo are contributed equally to these work

'Shanxi Key Laboratory of Otorhinolaryngology Head and Neck Cancer, First

Hospital of Shanxi Medical University, Taiyuan 030001, China

${ }^{2}$ Shanxi Province Clinical Medical Research Center for Precision Medicine of

Head and Neck Cancer, First Hospital of Shanxi Medical University, Taiyuan 030001, China

Full list of author information is available at the end of the article
}

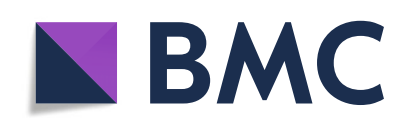

(c) The Author(s). 2020 Open Access This article is licensed under a Creative Commons Attribution 4.0 International License, which permits use, sharing, adaptation, distribution and reproduction in any medium or format, as long as you give appropriate credit to the original author(s) and the source, provide a link to the Creative Commons licence, and indicate if changes were made. The images or other third party material in this article are included in the article's Creative Commons licence, unless indicated otherwise in a credit line to the material. If material is not included in the article's Creative Commons licence and your intended use is not permitted by statutory regulation or exceeds the permitted use, you will need to obtain permission directly from the copyright holder. To view a copy of this licence, visit http://creativecommons.org/licenses/by/4.0/. The Creative Commons Public Domain Dedication waiver (http://creativecommons.org/publicdomain/zero/1.0/) applies to the data made available in this article, unless otherwise stated in a credit line to the data. 
(Continued from previous page)

Conclusion: Our study reveals that the novel autophagy-suppressive circPARD3 promotes LSCC progression and chemoresistance through the PRKCI-Akt-mTOR pathway, providing new insights into circRNA-mediated autophagy regulation and potential biomarker and target for LSCC treatment.

Keywords: Autophagy, Circular RNA, Chemoresistance, PRKCI, Migration and invasion, PI3K-Akt-mTOR signaling pathway

\section{Background}

Laryngeal squamous cell carcinoma (LSCC) is the second most common malignant tumor in head and neck, with increasing incidence and mortality [1]. LSCC is prone to local invasion, cervical lymph node metastasis and chemoresistance, which are the main factors leading to poor prognosis in patients [2,3]. Autophagy is the biological process of eukaryotic cells using lysosomes to degrade their damaged organelles and macromolecular substances. Autophagy is important for cellular metabolism, organelles renewal and intracellular homeostasis maintenance [4]. Recent studies suggested that autophagy plays pivotal role in tumorigenesis, recurrence, metastasis, and chemoresistance [5]. However, the autophagy status and regulatory mechanisms in LSCC remain unclear.

Circular RNAs (circRNAs) are a class of covalently closed circular RNA molecules, which are more stable than linear RNA because they are unaffected by RNA exonuclease. Therefore, circRNAs have unique advantages as markers for disease diagnosis and prognosis [6]. circRNA expression has tissue and cell specificity, playing an important regulatory role in various physiological and pathological processes [7]. circRNAs can function as a microRNA (miRNA) sponge to bind miRNAs specifically. In consequence, it relieves the inhibitory effects of miRNA on downstream target genes, thus upregulating the expression levels of the target genes [8]. Furthermore, some circRNAs exert biological functions via binding to RNA-binding proteins or translating into proteins $[9,10]$. Previous studies indicated that circRNAs are involved in regulation of autophagy in several diseases such as breast cancer, sciatic nerve injury, and cerebral ischemic stroke [11-13]. However, the regulatory relationship between circRNAs and autophagy and the clinical significance of autophagy-related circRNAs in diagnosis and treatment of LSCC remain largely unexplored.

In this study, we demonstrated that autophagy is inhibited in LSCC. Strikingly, we identified a novel autophagy suppressive circRNA circPARD3, which is significantly upregulated in LSCC tissues. High circPARD3 expression is significantly associated with malignant progression and poor prognosis of LSCC patients. We found that $\operatorname{circPARD} 3$ promotes LSCC cell proliferation, migration, invasion and chemoresistance by inhibiting autophagy. Our data further revealed that $\operatorname{circPARD} 3$ upregulates PRKCI expression by sponging $m i R-145-5 p$, thereby activating the Akt-mTOR signaling axis and inhibiting autophagy. These findings extend the understanding of autophagy and circRNA in LSCC progression and chemoresistance, and highlight the significance of circPARD3 in the regulation of autophagy and chemoresistance in LSCC.

\section{Methods}

\section{Tumor specimens}

Tumor specimens were collected from patients undergoing surgery at the Department of Otolaryngology Head and Neck Surgery, First Hospital of Shanxi Medical University. A total of three cohorts of LSCC specimens were used in this study (Additional file 3: Figure S1). Cohort 1 of 138 LSCC patients with available archived formalinfixed paraffin-embedded (FFPE) LSCC tissues was used for immunohistochemical staining (IHC) or immunofluorescence (IF). Cohort 2 of 107 LSCC cases with LSCC tissues $(n=107)$ and paired adjacent normal mucosa (ANM) tissues $(n=107)$ was used for RNA sequencing. Cohort 3 of 100 LSCC cases with LSCC tissues and paired ANM tissues was used for qPCR experiments. None of the patients received chemotherapy or radiotherapy before surgery. Clinical features of LSCC samples are shown in Additional file 1: Table S1-3.

\section{Antibodies and reagents}

Antibodies against to Akt (Cat \# 4685S), Phospho-Akt (Thr308) (Cat \# 2965S), Phospho-Akt (Ser473) (Cat \# 4060S), mTOR (Cat \# 2983S), Phospho-mTOR (Ser2448) (Cat \# 5536S), LC3B (Cat \# 3868S), SQSTM1/ p62 (Cat \# 88588S), E-Cadherin (Cat \# 3195S), NCadherin (Cat \# 13116S), and Vimentin (Cat \# 5741S) were purchased from Cell Signaling Technology (Danvers, MA). PRKCI antibody (Cat \# 13883-1-AP) was purchased from Proteintech Group, Inc. (Rosemont, IL). GAPDH antibody (Cat \# HC301-02) was purchased from TransGen Biotech (Beijing, China). Rapamycin (Cat \# S1039), SC79 (Cat \# S7863), 3-Methyladenine (3MA) (Cat \# S2767), MK-2206 2HCl (MK-2206) (Cat \# S1078), MHY1485 (Cat \# S7811), and Actinomycin D (Cat \# S8946) were purchased from Selleck Chemicals 
(Houston, TX). Detailed information of the chemicals is shown in Additional file 1: Table S4.

\section{Cell culture}

Human LSCC cell line Tu 177 (purchased from Bioleaf Biotech Corporation, Shanghai, China), human oral keratinocytes (HOK) (obtained from ScienCell Research Laboratories, Carlsbad, CA), HEK293T cells (obtained from the China Center for Type Culture Collection), human LSCC cell line FD-LSC-1 (kindly provided by Professor Liang Zhou [14]), and human normal lung fibroblast cell line MRC-5 (purchased from the China Center for Type Culture Collection) were cultured in a humidified atmosphere at $37{ }^{\circ} \mathrm{C}$ with $5 \% \mathrm{CO} 2$. Cell lines were confirmed to be mycoplasma free using the TransDetect PCR Mycoplasma Detection Kit (TransGen Biotech, Beijing, China). For half-life analysis of RNA, transcription was inhibited using Actinomycin D $(2 \mu \mathrm{g} /$ $\mathrm{mL}$ ). Detailed information for cell lines and media is shown in Additional file 1: Table S5.

\section{RNA and genomic DNA (gDNA) extraction, RNase R treatment, and quantitative real time PCR (qPCR)}

Total RNA was extracted from cells and tumor samples using Trizol reagent (Invitrogen, Waltham, MA) according to the manufacturer's instructions. Cytoplasmic and nuclear RNA was isolated using a PARIS kit (ThermoFisher Scientific, Waltham, MA). gDNA was extracted using a genomic DNA isolation kit (TIANGEN Biotech (Beijing) Co., Ltd., Beijing, China). For RNase R treatment, $2 \mu \mathrm{g}$ of total RNA was incubated for $10 \mathrm{~min}$ at $37^{\circ} \mathrm{C}$ with or without $3 \mathrm{U} / \mu \mathrm{g}$ RNase R (Geneseed Biotech Co., Ltd., Guangzhou, China). For PCR or qPCR analysis of mRNA and circRNA, cDNA was synthesized using a HiScript II 1st Strand cDNA Synthesis Kit (Vazyme, Nanjing, China). For qPCR of miRNA, cDNA was synthesized using an All-in-One miRNA FirstStrand cDNA Synthesis Kit (GeneCopoeia, Rockville, MD). qPCR was performed using PerfectStart Green qPCR SuperMix (TransGen Biotech) on LightCycler 96 Instrument (Roche, Basel, Switzerland). The relative RNA expression levels were calculated using the 2 $(-\triangle \triangle \mathrm{CT})$ method. The $18 \mathrm{~s}$ rRNA (for circRNA and mRNA) and U6 small nuclear RNA were used as internal controls. Primer sequences are shown in Additional file 1: Table S6.

\section{RNA sequencing}

The RNA integrity was examined with Bioanalyzer 2100 (Agilent, Santa Clara, CA). The RNA samples of 107 LSCC and paired ANM tissue with RIN $>7$ were subjected to library construction, and then sequenced on Illumina HiSeq 4000 (circRNA and mRNA) or HiSeq 2000 (miRNA). RNA sequencing technology was provided by Novogene (Beijing, China). Differentially expressed circRNAs, miRNAs, and mRNAs were analyzed as previous reported [15] (Additional file 1: Table S7-9).

\section{Plasmid construction}

The circPARD3 overexpression plasmid (circPARD3-OE) was generated by inserting the full-length of circPARD3 sequence into the pLO5-ciR lentiviral vector (Geneseed Biotech Co., Ltd). shRNA lentiviral plasmid targeting circPARD3 (sh-circPARD3) was constructed by inserting annealed shRNA template DNA sequence into the pLKO.1 vector. The PRKCI overexpression plasmid (PRKCI-OE) was generated by inserting the PRKCI ORF sequence into the pLenti-puro vector (a gift from Ie-Ming Shih, Addgene plasmid \# 39481 [16]). To construct luciferase reporter plasmids, the sequences of wild-type circPARD3, miR-145-5p binding site mutated $\operatorname{circPARD3,}$ wild-type $P R K C I$ 3' UTR, and miR-145-5p binding site mutated PRKCI 3' UTR were cloned into the psiCHECK2 vector (Promega, Madison, WI), respectively.

siRNAs, miRNA mimics, and inhibitor

siRNAs targeting circPARD3 (si-circ-1: sense: 5'-CACCUG UAGACAGGUAAAUAC-3', antisense: 5' - GUAUUUACC UGUCUACAGGUG-3'; si-circ-2: sense: 5' -CUGUAGACAG GUAAAUACCAG-3', antisense: 5' - CUGGUAUUUACCU GUCUACAG-3'), siRNAs targeting PRKCI (si-PRKCI: sense: 5'-CCGGGUAAUAGGAAGAGGAAGUUAU-3', antisense: 5'-AUAACUUCCUCUUCCUAUUACCCGG-3'), negative control siRNAs (si-NC), miR-145-5p mimics, miR-145-5p inhibitor, and NC mimics/inhibitor were synthesized by GenePharma (Shanghai, China). RNA molecule was modified by 2'-OMe to enhance stability. Transfection efficiency of siRNAs, miRNA mimics, and inhibitor was verified by transfecting cells with Cy3 labeled negative control siRNAs (si-NC$\mathrm{Cy} 3$ ), miRNA mimics (NC mimics-Cy3), and inhibitor (NC inhibitor-Cy3) (Additional file 3: Figure S2).

\section{Screening of autophagy related circRNA by high-content platform based autophagic flux analysis}

To screen autophagy related circRNAs in LSCC, siRNAs targeting the top 15 upregulated circRNAs in the LSCC samples compared to their paired ANM tissues were designed and synthesized by GenePharma (Shanghai, China). FD-LSC-1 and Tu 177 cells stably expressing LC3B-GFP were transfected with siRNAs or si-NC for $24 \mathrm{~h}$, then cells were seeded into 48 -well plates and incubated for additional $24 \mathrm{~h}$. Cells were stained with DAPI and were imaged on a PerkinElmer Operetta CLS highcontent analysis system (Waltham, MA). Autophagy level was assessed by comparing the amount of green LC3 dots between NC group and specific circRNA knockdown groups. 


\section{Autophagic flux detection in cells}

A tandem mCherry-EGFP-tagged LC3B was used to monitor autophagic flux in cells as previous reported [17]. Briefly, FD-LSC-1 and Tu 177 cells were transduced with mCherry-EGFP-LC3B lentiviruses for $48 \mathrm{~h}$, then mCherry-EGFP-LC3B expressing cells were screened by puromycin. For autophagic flux detection, mCherry-EGFP-LC3B expressed cells were examined by Leica TCS SP8 confocal laser scanning microscope
(Leica Microsystems Inc.). The number of GFP and mCherry puncta per cell was quantified manually. At least 10 cells in each of three independent experiments were analyzed randomly.

\section{Bioinformatics analysis}

Gene Set Enrichment Analysis (GSEA) [18] was conducted to explore differentially expressed mRNA involved gene sets. Kyoto Encyclopedia of Genes and

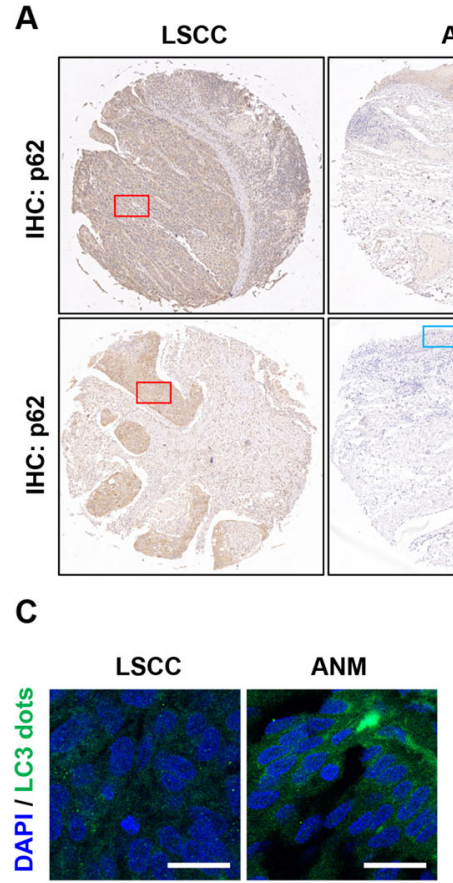

A

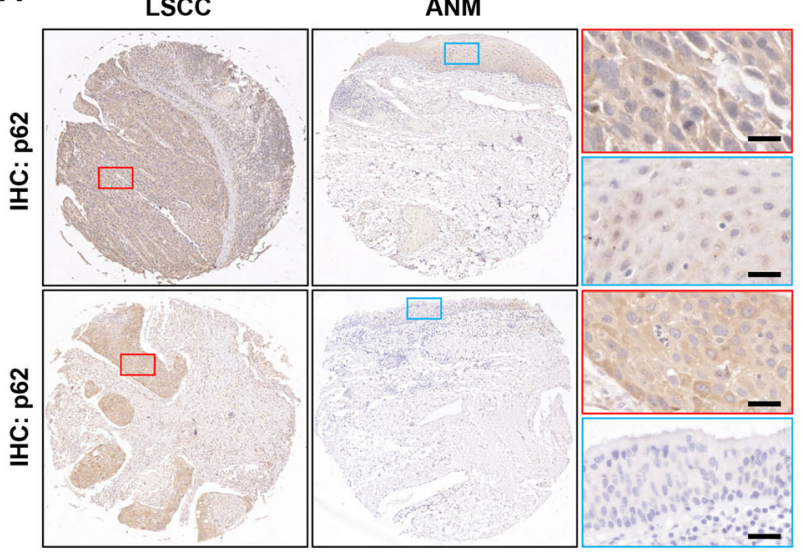

D

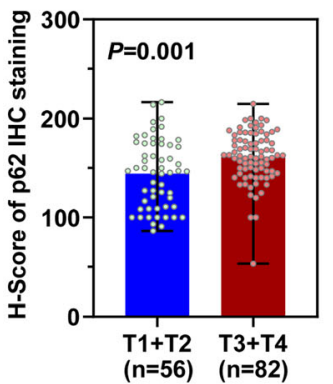

B

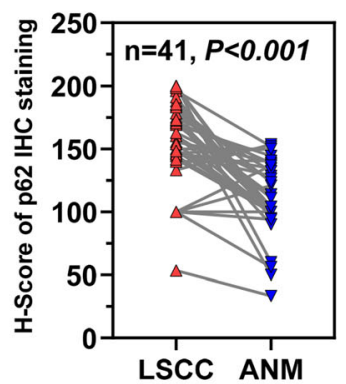

E

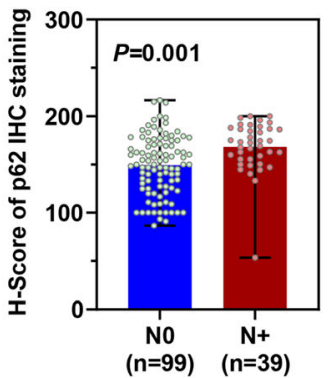

$\mathbf{F}$

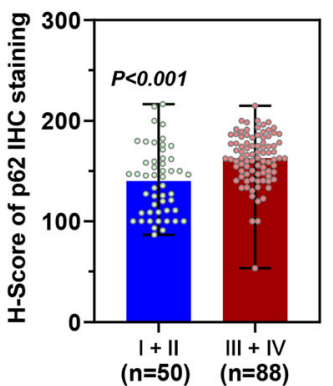

G

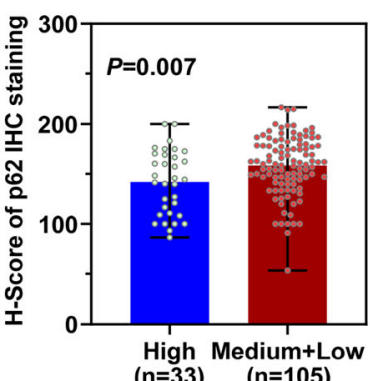

$\mathrm{H}$

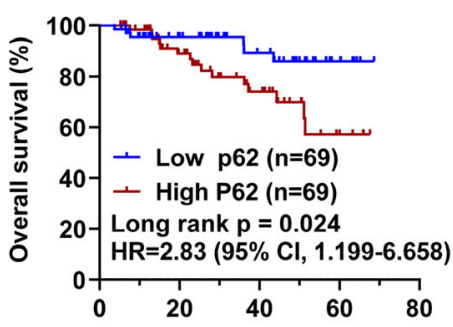

Fig. 1 High p62 level is associated with malignant progression and poor prognosis of LSCC patients. Protein levels of p62 in 138 LSCC tissues and 41 ANM tissues (Cohort 1) were determined by IHC staining. a Representative image of p62 IHC staining in LSCC tissues and paired ANM tissues. Scale bar, $20 \mu \mathrm{m}$. b IHC staining results of p62 in 41 paired LSCC tissues and ANM tissues were quantified with CaseViewer version 2.3 and presented as H-Score. c Representative confocal microscopy images of LC3B dots (green) in formalin-fixed paraffin-embedded LSCC tissues and paired ANM tissues, nuclei were stained with DAPI (blue). Scale bar, $20 \mu \mathrm{m}$. $\mathbf{d}-\mathbf{g}$ Differential expression of p62 protein in 138 LSCC tissues with different T stages (d), N stages (e), clinical stages $(\mathbf{f})$, and pathological differentiation degrees $(\mathbf{g})$. The difference of H-score of p62 between two groups was analyzed by Two-tailed Student's t-test. $\mathbf{h}$ Kaplan-Meier analysis of the association between p62 protein levels and overall survival in 138 LSCC patients. Expression levels of p62 were divided into high and low subgroups according to the median $\mathrm{H}$-score of IHC staining 
Genomes (KEGG) pathway analysis was performed using the clusterProfiler [19] to identify differentially expressed mRNA enriched signaling pathways. Target gene prediction of $h s a-m i R-145-5 p$ was performed using the Targetminer program [20] (http://www.isical.ac.in/ bioinfo_ $\mathrm{miu} /$ targetminer20.htm). circPARD3 binding miRNA was predicted using the CircInteractome program [21] (https://circinteractome.nia.nih.gov/mirna_target_sites.

html).

\section{circRNA pulldown}

circRNA pulldown was performed using Biotin-labeled circPARD3 probes (Sangon Biotech, Shanghai, China). Briefly, FD-LSC-1 cells were lysed by IP lysis buffer
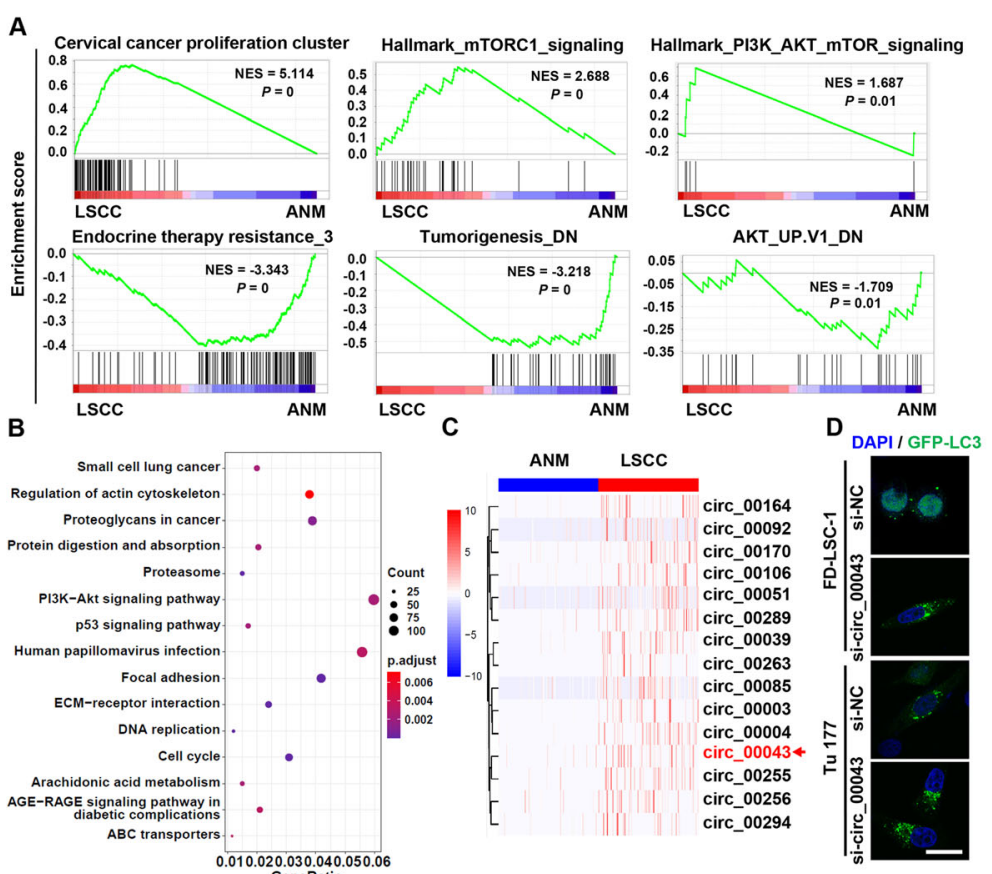

E 0.010 .020 .030 .040 .050 .06 $\mathbf{F}$

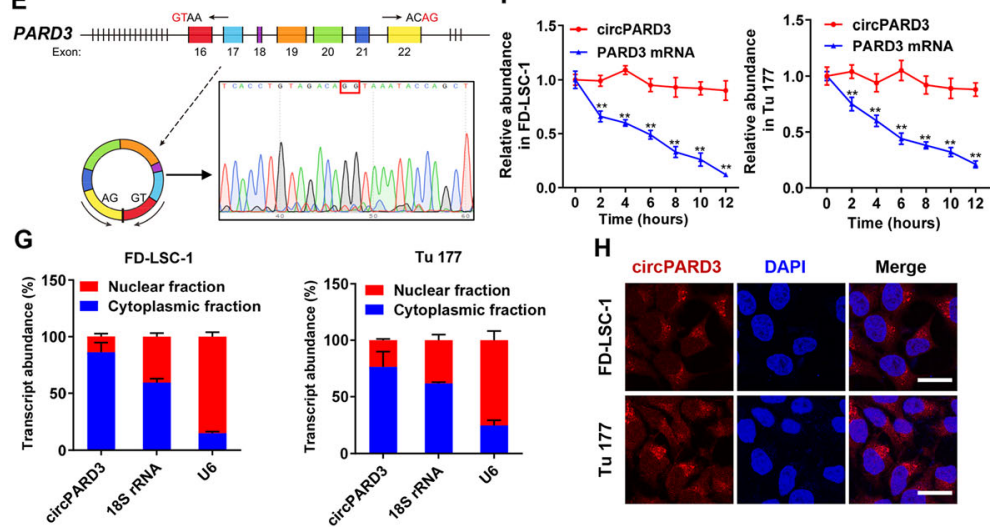

Fig. 2 Identification of the autophagy suppressive circRNA circPARD3 in LSCC. a-c RNA sequencing was performed on 107 LSCC tissues and paired ANM tissues (Cohort 2) to screen differentially expressed circRNAs, mRNAs, and miRNAs. GSEA analysis of differentially expressed mRNAs (a). NES, normalized enrichment score. KEGG pathway analysis of differentially expressed mRNAs (b). Top 15 pathways were plotted according to enriched gene ratio and $p$ value. $\mathbf{c}$ Hierarchical clustering of circRNA differential expression profiles between 107 LSCC tissues and 107 paired ANM tissues. The heatmap was generated from the top 15 differentially expressed circRNAs. d FD-LSC-1 and Tu 177 cells expressing LC3B-GFP were transfected with siRNAs or si-NC for $24 \mathrm{~h}$, then cells were seeded into 48 well plates and incubated for additional $24 \mathrm{~h}$. LC3 dots were analyzed on high-content screening system. Representative images of si-circ_00043 transfected cells were shown. Scale bar, 20 mm. e Schematic diagram of the genomic location and splicing pattern of circPARD3 is shown (Left). Validation of the head-to-tail splicing of circPARD3 by RT-PCR and Sanger sequencing (Right). f The relative RNA levels of cirCPARD3 and linear PARD3 mRNA in FD-LSC-1 and Tu 177 cells treated with actinomycin D at the indicated time points were determined by qPCR. $\mathbf{g}$ circPARD3 levels in cytoplasm and nucleus of FD-LSC-1 and Tu 177 cells were determined by qPCR analysis. $18 \mathrm{~S}$ and U6 RNA were used as positive controls of in the cytoplasm and nucleus, respectively. $\mathbf{h}$ RNA fluorescence in situ hybridization for circPARD3 was performed in FD-LSC-1 and Tu 177 cells. Nuclei were stained with DAPI (blue) and circPARD3 probes were labeled with Cy3 (red). Scale bar, $20 \mu \mathrm{m}$. Error bars ( $\mathbf{f}$ and $\mathbf{g}$ ) represent SD of three independent experiments. ${ }^{*} P<0.01$ 
supplemented with $1 \times$ protease inhibitor and $1 \mathrm{U} / \mu \mathrm{L}$ RNase inhibitor (ThermoFisher Scientific, Waltham, MA) on ice for $30 \mathrm{~min}$ with occasional mixing. The lysate was sonicated for 2 min with $10 \mathrm{~s}$ on/off cycles. Then the lysate was precleared with $30 \mu \mathrm{L}$ streptavidin agarose beads (Cat \# S1638, Merck KGaA, Darmstadt, Germany) for $1 \mathrm{~h}$ at $4{ }^{\circ} \mathrm{C}$ with rotation. Biotinlabeled $\operatorname{circPARD} 3$ probes or the negative control $(\mathrm{NC})$ probes were added to the precleared lysate, and the mixtures were incubated at $4{ }^{\circ} \mathrm{C}$ overnight. Next, the mixture was incubated with $30 \mu \mathrm{L}$ streptavidin agarose beads at $37^{\circ} \mathrm{C}$ for $1 \mathrm{~h}$. Wash beads-probeprotein complex with $1 \mathrm{~mL}$ IP lysis buffer for six times, and then total RNA bound to the beads was extracted, followed by reverse transcription and qPCR detection of $\operatorname{circPARD} 3$ and miRNAs.

\section{Xenograft tumorigenesis}

Animal experiments were approved by the Animal Experimental Research Ethics Committee of Shanxi
A

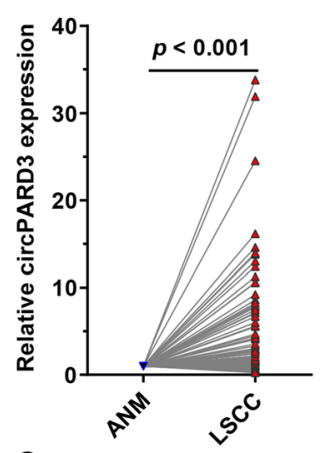

C

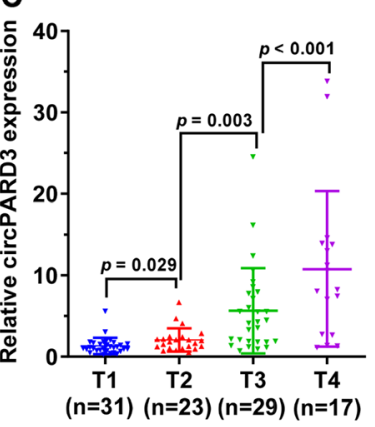

$\mathbf{F}$

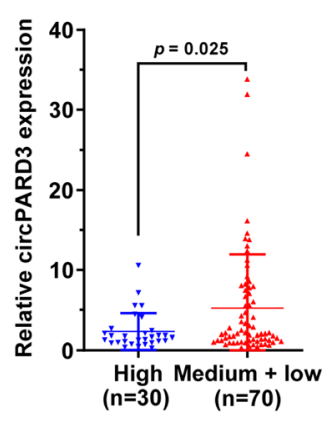

B

G

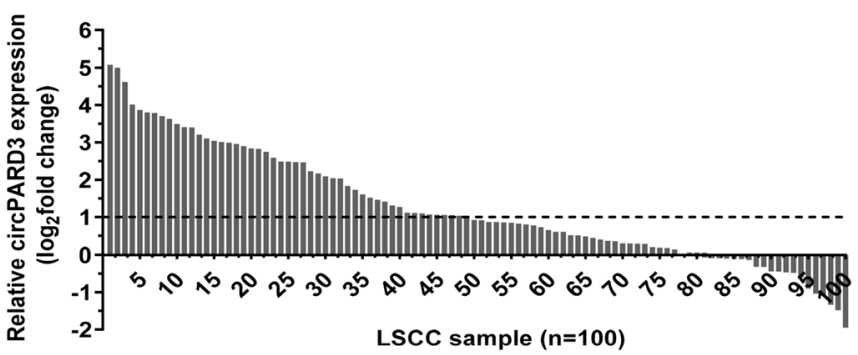

D
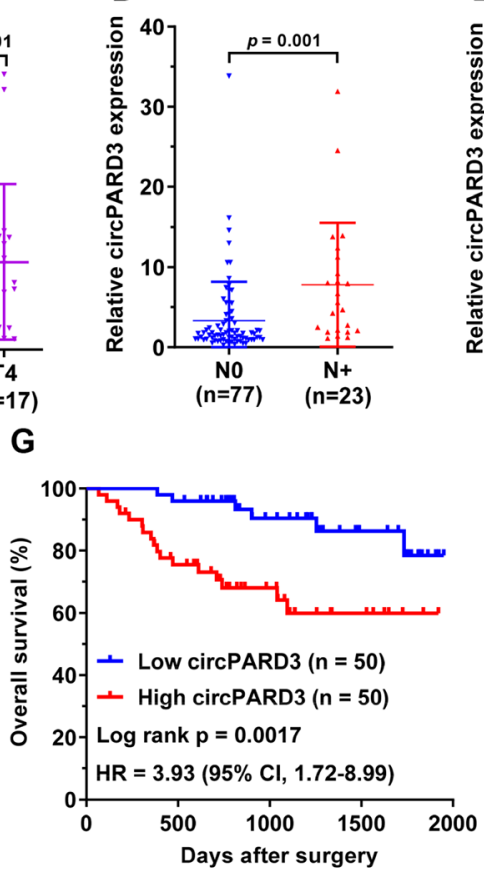

E
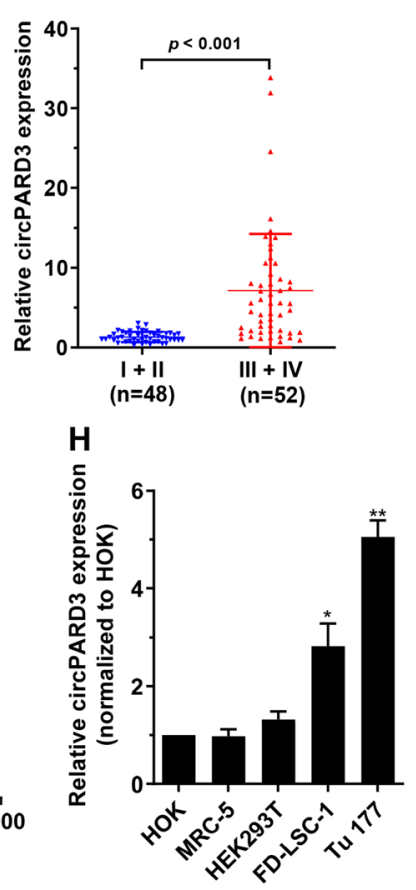

Fig. 3 Upregulation of cirCPARD3 and its clinical significance in LSCC. The relative RNA levels of cirCPARD3 in 100 LSCC tissues and paired ANM tissues (Cohort 3) were determined by qPCR. a Comparison of circPARD3 levels between LSCC tissues and paired ANM tissues $(n=100)$. Twotailed Student's t-test. $\mathbf{b}$ The relative expression levels of circPARD3 in 100 LSCC tissue samples were normalized to paired ANM tissues. c-f Association analysis between circPARD3 levels and T stages (c), N stages (d), clinical stages (e), and pathological differentiation degrees (f). The difference of circPARD3 levels between two groups was analyzed by Two-tailed Student's t-test. Error bars represent SD. g Kaplan-Meier analysis of the association between circPARD3 levels and overall survival of 100 LSCC patients. Expression levels of circPARD3 were divided into high and low subgroups according to the median relative RNA abundance of $\mathrm{QPCR}$ experiments. $\mathbf{h}$ The relative expression of circPARD3 in LSCC cell lines (FD-LSC-1, Tu 177) and normal control cell lines (HOK, MRC-5, HEK293T) was determined by qPCR analysis. Error bars represent SD of three independent experiments. ${ }^{*} P<0.05,{ }^{*} P<0.01$ 
Medical University and conducted according to its guidelines. BALB/C nude mice (female, 6 weeks) were obtained from Vital River Laboratory Animal Technology Co., Ltd. (Beijing, China) and housed in isolation and ventilation cages (TECNIPLAST S.p.A., Italy) under SPF (Specific pathogen Free) conditions in a climatecontrolled room $\left(25 \pm 1.5^{\circ} \mathrm{C}\right)$ with $12 \mathrm{~h}$ light/dark illumination cycle and $50 \pm 10 \%$ humidity. To construct xenograft tumor models, $200 \mu \mathrm{L}$ serum-free DMEM containing $1 \times 10^{7}$ FD-LSC-1 cells were subcutaneously injected into the right flank of each mouse. From day 13, mice were randomized into two groups and intraperitoneally injected with $1 \mathrm{mg} / \mathrm{kg}$ Cisplatin (CDDP dissolved in normal Saline, $100 \mu \mathrm{L}$ ) or equal volume of normal Saline (NS) every 2 days. Tumor volume was calculated using the following formula: $\mathrm{V}$ (volume) $=$ (length $\times$ width $\left.^{2}\right) / 2$. Mice were sacrificed at day 33 and tumors were dissected, weighed, and resected for qPCR, HE staining, IHC staining, and immunofluorescence analysis.
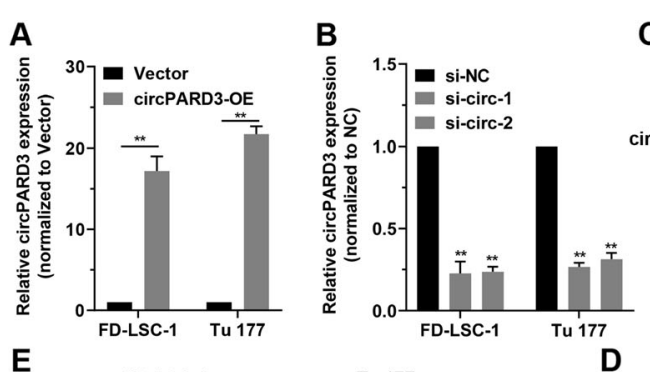

C

E

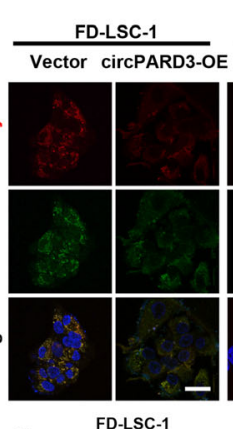

Tu 177
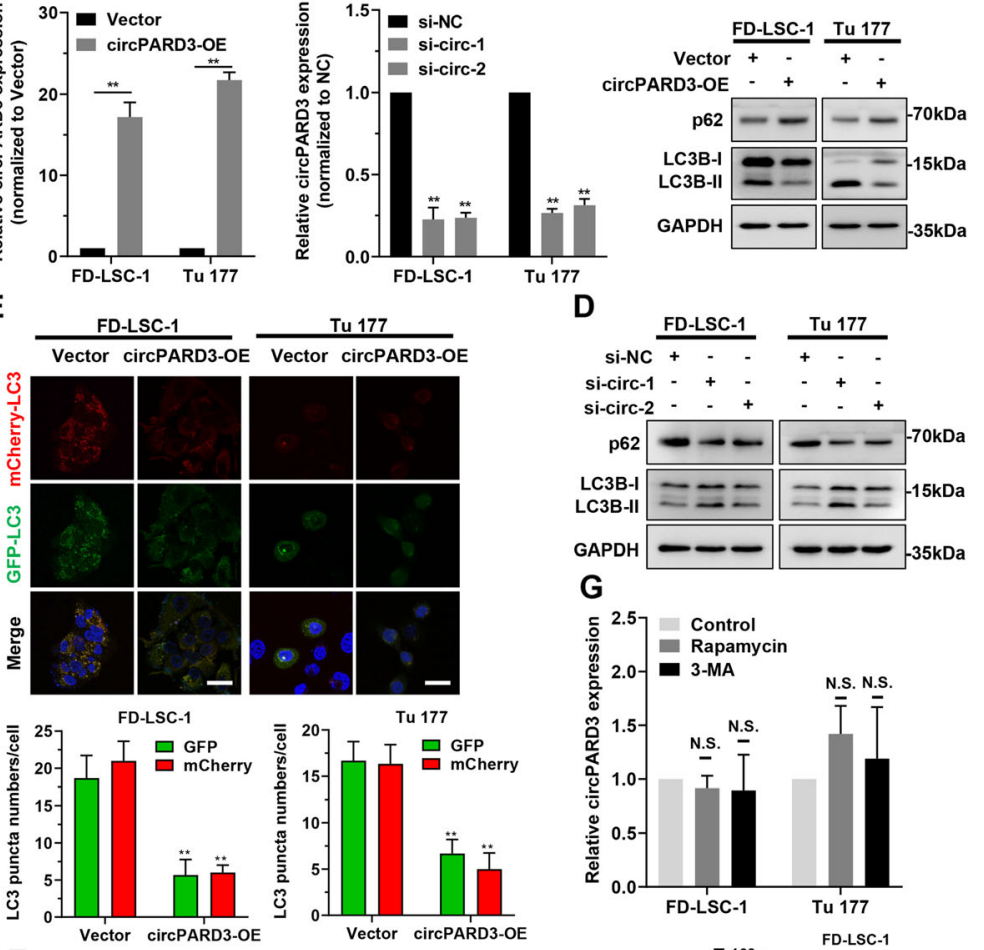

$\mathbf{F}$
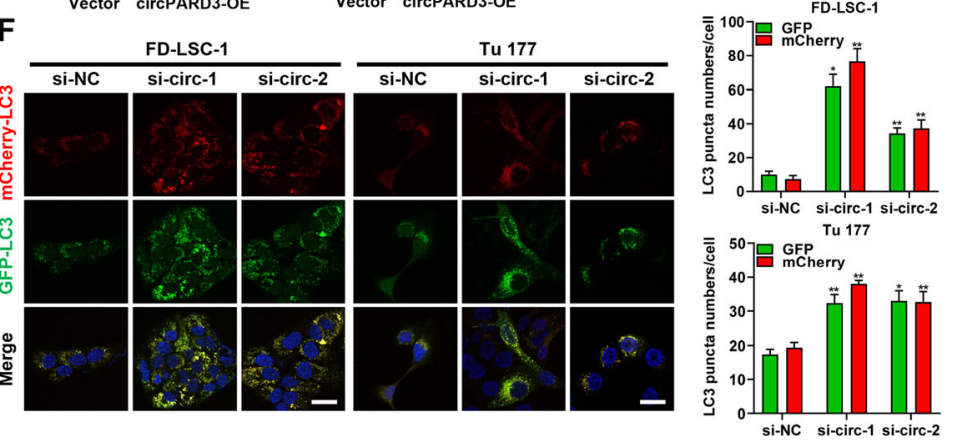

Fig. 4 cirCPARD3 inhibits autophagy of LSCC cells. a FD-LSC-1 and Tu 177 cells were infected with circPARD3 overexpression lentiviruses (circPARD3-OE) or control empty vector (Vector). Expression of circPARD3 was examined by qPCR. $\mathbf{b}$ FD-LSC-1 and Tu 177 cells were transfected with siRNAs targeting circPARD3 (si-circ-1 and si-circ-2) or NC siRNAs (si-NC) for $48 \mathrm{~h}$. Expression of circPARD3 was examined by qPCR. $\mathbf{c}$ and $\mathbf{d}$ FDLSC-1 and Tu 177 cells were infected with circPARD3 overexpression lentiviruses or transfected with siRNAs targeting circPARD3. Western blotting was performed to detect the expression of LC3B and p62 after overexpression (c) or silencing of circPARD3 (d). e and $\mathbf{f}$ mCherry-EGFP-LC3B labeled FD-LSC-1 and Tu 177 cells were infected with cirCPARD3 overexpression lentiviruses or transfected with siRNAs targeting circPARD3. Autophagic flux was analyzed by confocal microscopy after overexpression (e) or silencing (f) of circPARD3. Scale bar, $25 \mu \mathrm{m}$. $\mathbf{g}$ qPCR analysis of circPARD3 in FD-LSC-1 and Tu 177 cells after Rapamycin (100 nM for $24 \mathrm{~h}$ ) or 3-Methyladenine (3-MA, $2.5 \mathrm{mM}$ for $24 \mathrm{~h}$ ) treatment. Error bars represent SD of three independent experiments. ${ }^{*} P<0.05$, ${ }^{* *} P<0.01$. N.S., no significant 
More detailed information of methods could be obtained in Additional file 2: Supplemental Materials and Methods.

\section{Statistical analysis}

Statistical analysis was performed using GraphPad Prism 8 software (San Diego, CA). Comparisons between two groups were conducted by the two-tailed Student's t-test. Kaplan-Meier method was used to analyze the survival probability of LSCC patients with different expression levels of p62 or circPARD3. Data were reported as mean $\pm \mathrm{SD}$ (standard deviation). $P$ values of $<0.05$ were considered statistically significant.

\section{Results}

Autophagy inhibition is associated with malignant progression and poor prognosis of LSCC

p62 (also known as SQSTM1) is an autophagic substrate that is degraded when autophagy is activated and accumulates when autophagy is decreased or inhibited [22]. To determine the autophagy levels of LSCC, we compared p62 protein expression level between LSCC tissues and paired adjacent normal mucosal (ANM) tissues via IHC

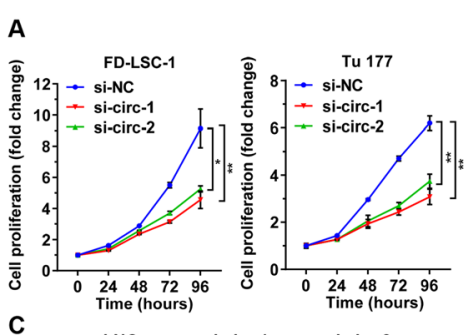

C

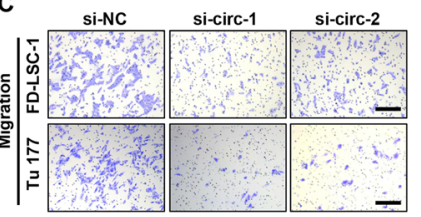

D

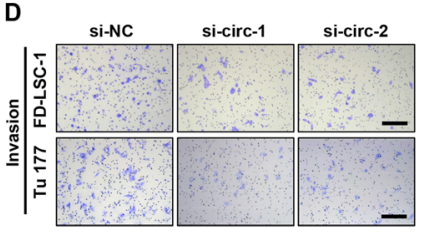

$\mathbf{F}$

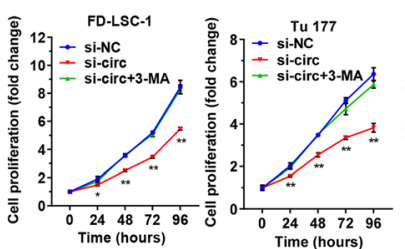

G
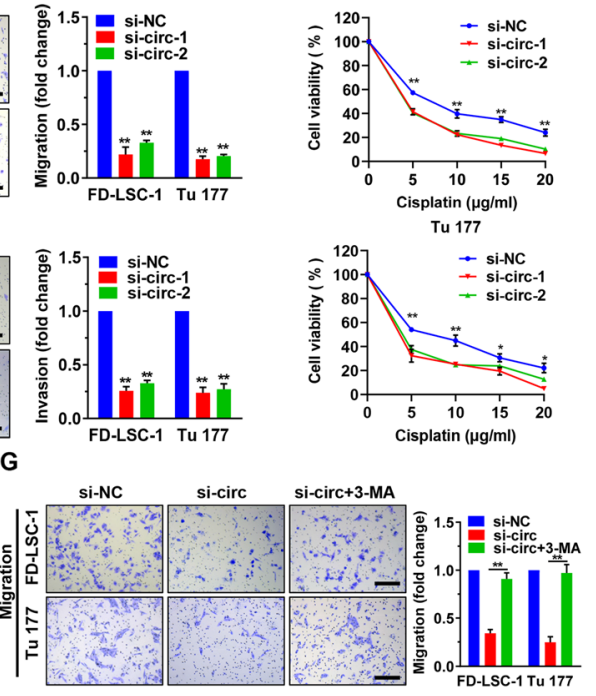

H
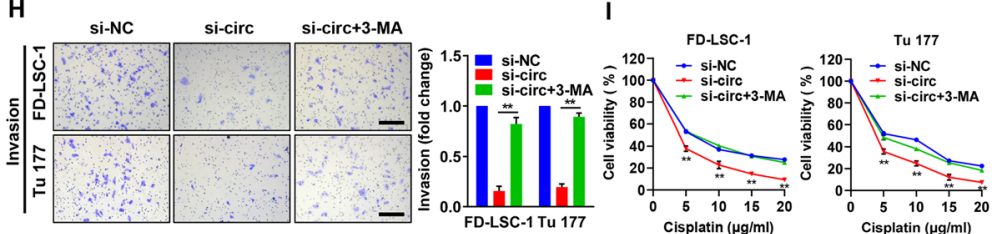

Fig. 5 cirCPARD3 promotes LSCC cell proliferation, migration, invasion and chemoresistance via inhibiting autophagy. a and $\mathbf{b}$ FD-LSC-1 and Tu 177 cells were transfected with siRNAs targeting circPARD3 (si-circ-1, si-circ-2) or si-NC, then cell proliferation was determined by CCK8 (a) and colony formation assays (b). c and $\mathbf{d}$ The migration (c) and invasion (d) abilities of FD-LSC-1 and Tu 177 cells after silencing of circPARD3 were detected by Transwell assays. Scale bar, 200 mm. e FD-LSC-1 and Tu 177 cells were transfected with siRNAs targeting circPARD3 or si-NC for 24 h, and then treated with various concentration of Cisplatin for another $24 \mathrm{~h}$. Cell viability was detected by CCK8 assays. f FD-LSC-1 and Tu 177 cells were transfected with si-circPARD3 (si-circ) or si-NC for $24 \mathrm{~h}$, and then treated with 3-MA (2.5 mM). Cell proliferation was determined by CCK8 assays. g-i FD-LSC-1 and Tu 177 cells were transfected with si-CirCPARD3 or si-NC for $24 \mathrm{~h}$, then treated with 3-MA (2.5 mM) for additional $24 \mathrm{~h}$. The migration (g) and invasion (h) abilities of FD-LSC-1 and Tu 177 cells after various treatments were detected by Transwell assays. Scale bar, 200 Hm. Cell viability of LSCC cells treated with various concentrations of Cisplatin for $24 \mathrm{~h}$ was determined by CCK8 assays (i). Error bars represent SD of three independent experiments. ${ }^{*} P<0.05,{ }^{* *} P<0.01$ 
A

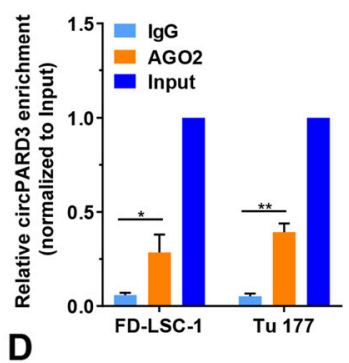

D

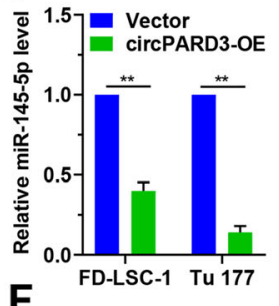

$\mathbf{F}$

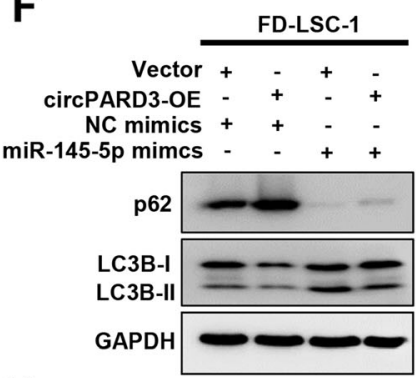

B
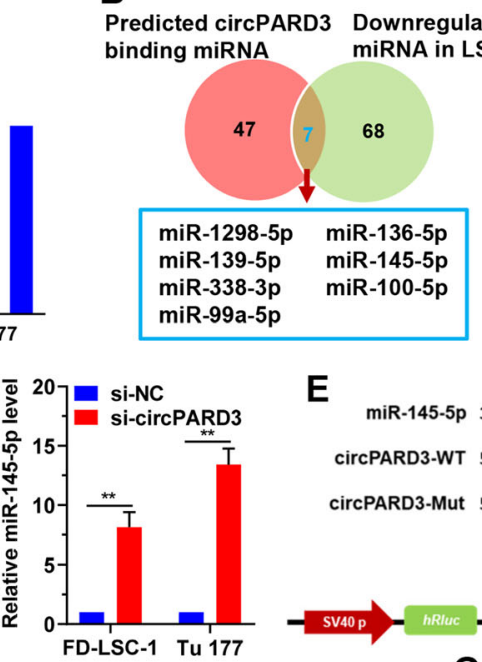

Tu 177

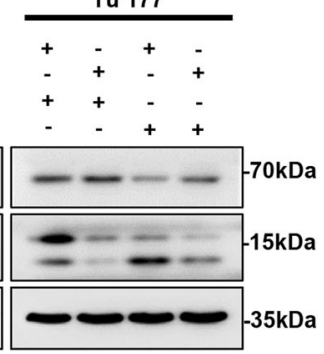

H
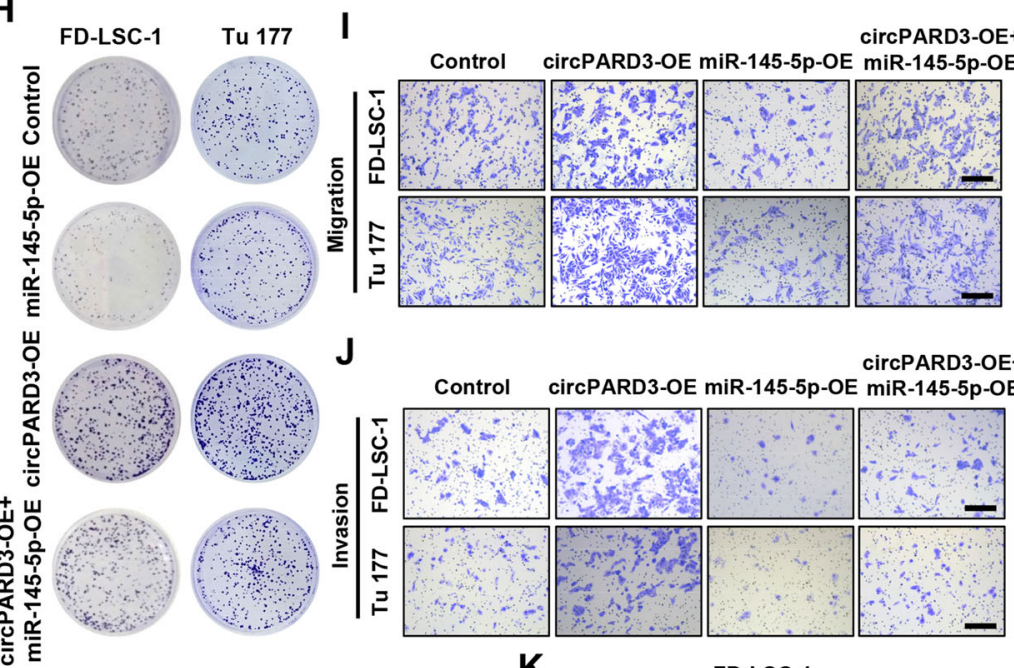

E

miR-145-5p $3^{\prime}$ UCCCUAAGGACCCUUUUGACCUG 5 ' circPARD3-WT 5' UAGAAAAAGGAUAAAACUGGAA $3^{\prime}$

circPARD3-Mut $5^{\prime}$ UAGAAAAAAGGAUAAUUGACCUA $3^{\prime}$

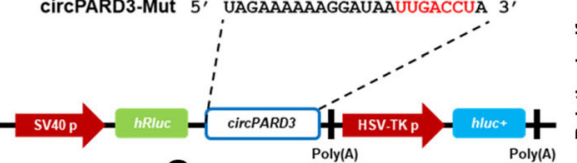

FD-LSC-1
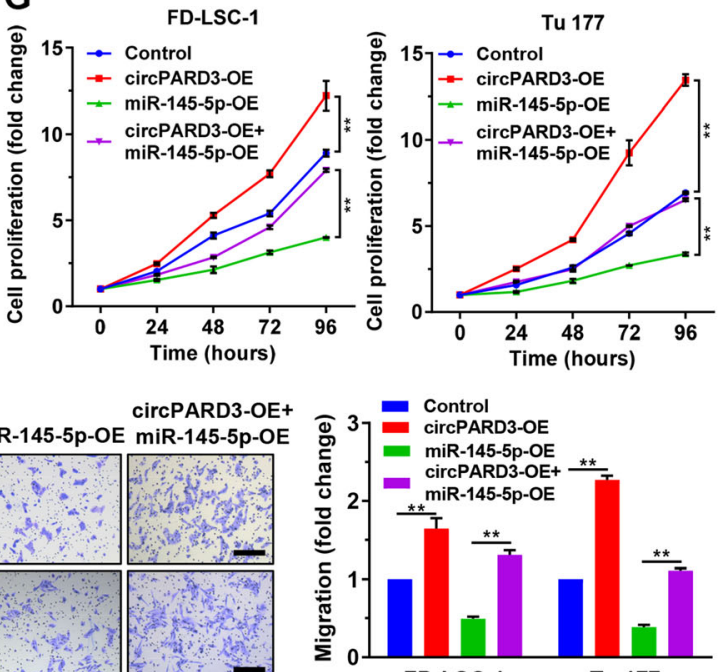

FD-LSC-1

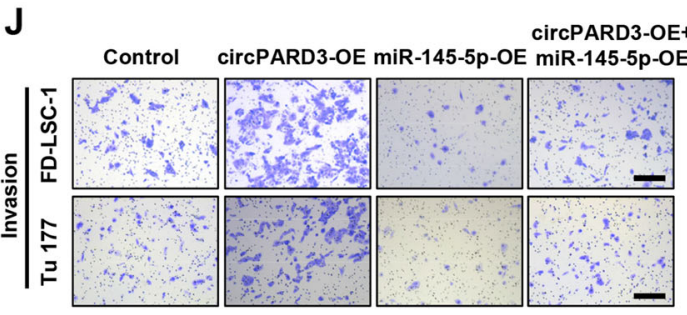

K
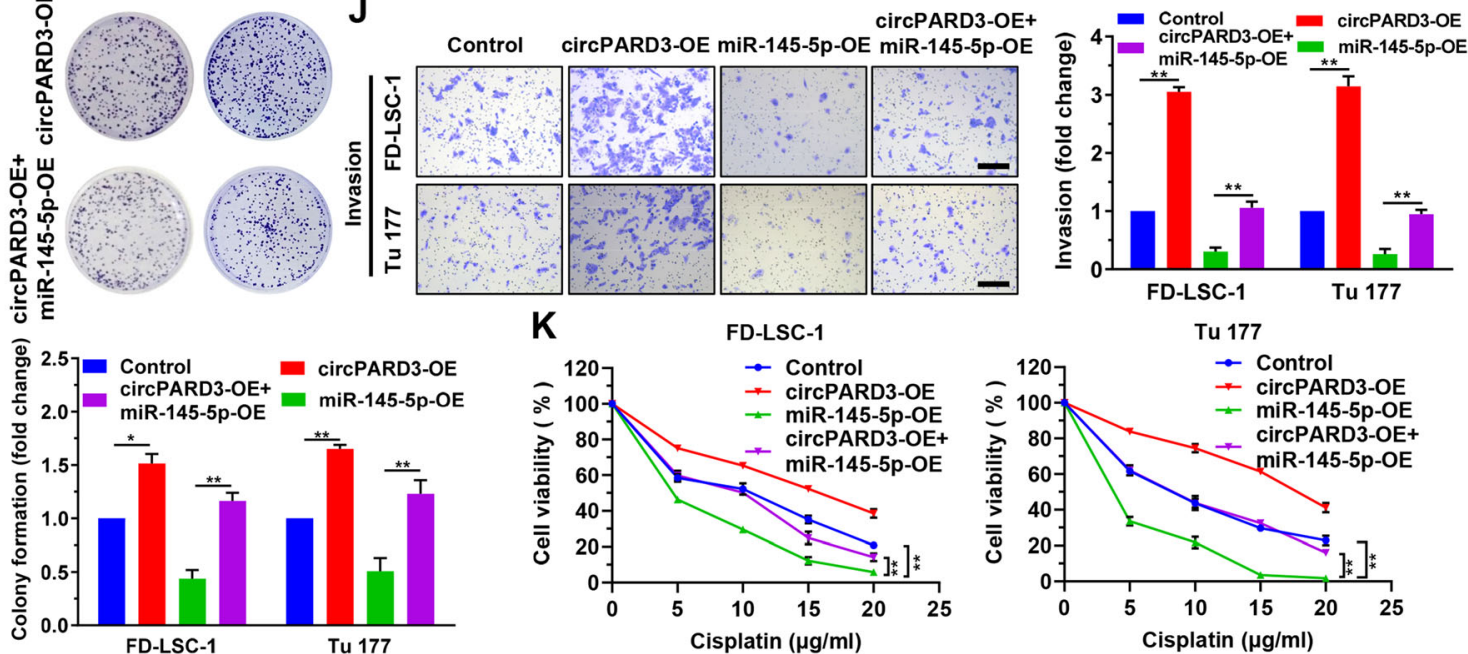

Tu 177

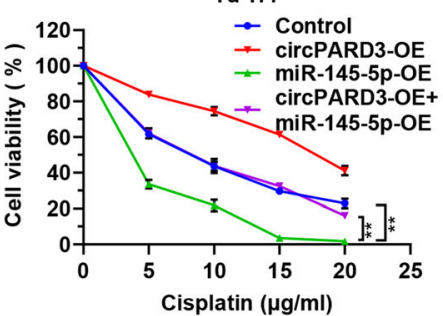

Fig. 6 (See legend on next page.) 
(See figure on previous page.)

Fig. 6 cirCPARD3 functions as a sponge of miR-145-5p in LSCC cells. a RIP assays were performed using an antibody against AGO2 with extracts from FD-LSC-1 and Tu 177 cells. Enrichment of circPARD3 in RNA samples after RIP assays was determined by qPCR analysis. b Venn diagram shows the intersection of predicted circPARD3 binding miRNAs and miRNAs downregulated in 107 LSCC tissues from the RNA sequencing data. c RNA pulldown assay was performed using Biotin-labeled circPARD3 probes in FD-LSC-1 cells, then the enrichment of the indicated miRNAs was detected by qPCR analysis. $\mathbf{d}$ Expression of miR-145-5p in LSCC cells overexpressing or silencing of circPARD3 was determined by qPCR analysis. e Luciferase reporter assay of FD-LSC-1 cells cotransfected with miR-145-5p mimics and luciferase reporter plasmid containing wild-type (WT) and miR-145-5p binding site mutated (Mut) circPARD3. N.S., no significant. f FD-LSC-1 and Tu 177 cells overexpressing circPARD3 (circPARD3-OE) were transfected with miR-145-5p mimics or NC mimics, expression of LC3B and p62 was detected by western blotting. $\mathbf{g}$ and $\mathbf{h}$ Cell proliferation of FD-LSC-1 and Tu 177 cells overexpressing circPARD3 and miR-145-5p was determined by CCK8 assays (g) and colony formation assays (h). The migration (i) and invasion (j) abilities of FD-LSC-1 and Tu 177 cells overexpressing circPARD3 and transfected with miR-145-5p mimics were evaluated by Transwell assays. Scale bar, $200 \mu \mathrm{m}$. $\mathbf{k}$ LSCC cells overexpressing circPARD3 and miR-145-5p mimics were treated with various concentrations of Cisplatin for $24 \mathrm{~h}$. Cell viability was determined by CCK8 assays. Error bars represent SD of three independent experiments. ${ }^{*} P<0.05,{ }^{* *} P<0.01$

staining. Results showed that p62 level in the LSCC tissues was significantly higher than that in the ANM tissues (Fig. 1a and b). Furthermore, immunofluorescence staining showed that LSCC tissues contained significantly fewer LC3 dots than that in ANM tissues (Fig. 1c). Analysis of the relationship between p62 level and clinicopathological parameters in 138 LSCC samples indicated that high p62 level was positively correlated with $T$ stages, cervical lymph node metastasis ( $\mathrm{N}$ stages) and clinical stages (Fig. 1d-f). Moreover, LSCC tissues with low and medium differentiation degree showed higher p62 level than that of their highly differentiated counteracts (Fig. 1g). KaplanMeier analysis showed that LSCC patients with high p62 level had shorter overall survival (Fig. 1h). Taken together, autophagy inhibition is an important molecular trait in LSCC and may play important roles in LSCC tumorigenesis and progression.

\section{Identification of the novel autophagy suppressive circRNA CirCPARD 3 in LSCC}

RNA sequencing on 107 LSCC tissues and paired ANM tissues found 304 differentially expressed circRNAs in the LSCC tissues, in which 189 upregulated and 115 downregulated (Additional file 1: Table S7). We also found 111 miRNAs with increased expression, and 75 miRNAs with decreased expression, 2467 mRNAs with increased expression, and 2140 mRNAs with decreased expression in LSCC tissues (Additional file 1: Table S8 and 9). GSEA analysis revealed that the differentially expressed genes were mainly involved in cancer cell proliferation, PI3K-Akt-mTOR, therapy resistance, and tumorigenesis (Fig. 2a). KEGG pathway analysis confirmed that the differentially expressed genes were mainly enriched in the PI3K-Akt, cell cycle, focal adhesion and other signaling pathways (Fig. 2b).

LC3B (Microtubule Associated Protein 1 Light Chain 3 Beta) is a recognized autophagic marker [23]. To identify autophagy related circRNAs, the top 15 circRNAs upregulated in LSCC tissues (Fig. 2c) were knockdown individually using siRNAs in LC3B-GFP expressing
LSCC cell lines FD-LSC-1 and Tu 177. High-content screening showed that LC3 dots were significantly increased in cells with circ_00043 knockdown (Fig. 2d), indicating that circ_00043 inhibited autophagy of LSCC cells. Sequence analysis indicated that circ_00043 is formed by backsplicing of exons 16-22 of human PARD3 mRNA (Fig. 2e), hence, we named it as circPARD3. The head-to-tail circular structure of circPARD3 was verified by RT-PCR and Sanger sequencing (Fig. 2e). Furthermore, RT-PCR using back-to-back primers demonstrated that the head-to-tail structure of circPARD3 was formed by backsplicing of the PARD3 mRNA rather than by genomic rearrangement (Additional file 3: Figure S3A).

LSCC cells were treated with actinomycin D, results showed that the half-life of circPARD3 was significantly longer than that of linear PARD3 mRNA (Fig. 2f). Furthermore, the total RNA purified from LSCC cells was treated with RNase $\mathrm{R}$, and we found that the linear PARD3 mRNA was decreased after RNase $\mathrm{R}$ treatment, while $\operatorname{circPARD} 3$ was not significantly degraded, indicating that $\operatorname{circPARD} 3$ is resistant to RNase R (Additional file 3: Figure S3B). After separating the nucleus and cytoplasm of FD-LSC-1 and Tu 177 cells, qPCR showed that circPARD3 mainly existed in the cytoplasm (Fig. $2 \mathrm{~g}$ ), which was further confirmed by RNA fluorescence in situ hybridization (Fig. 2h). Collectively, our study identified $\operatorname{circPARD} 3$ as an autophagy suppressive circRNA in LSCC, and we further verified its structural characteristics.

\section{Upregulation of circPARD3 is associated with malignant progression and poor prognosis of LSCC}

Next, we examined the expression level of circPARD3 in 100 LSCC tissues and paired ANM tissues. qPCR results showed that circPARD3 level in the LSCC tissues was significantly higher than that in the ANM tissues (Fig. 3a) in up to $80 \%$ of paired samples (Fig. $3 \mathrm{~b}$ ). Furthermore, high circPARD3 level was positively correlated with $\mathrm{T}$ stags, cervical lymph node metastasis ( $\mathrm{N}$ stages) and clinical 


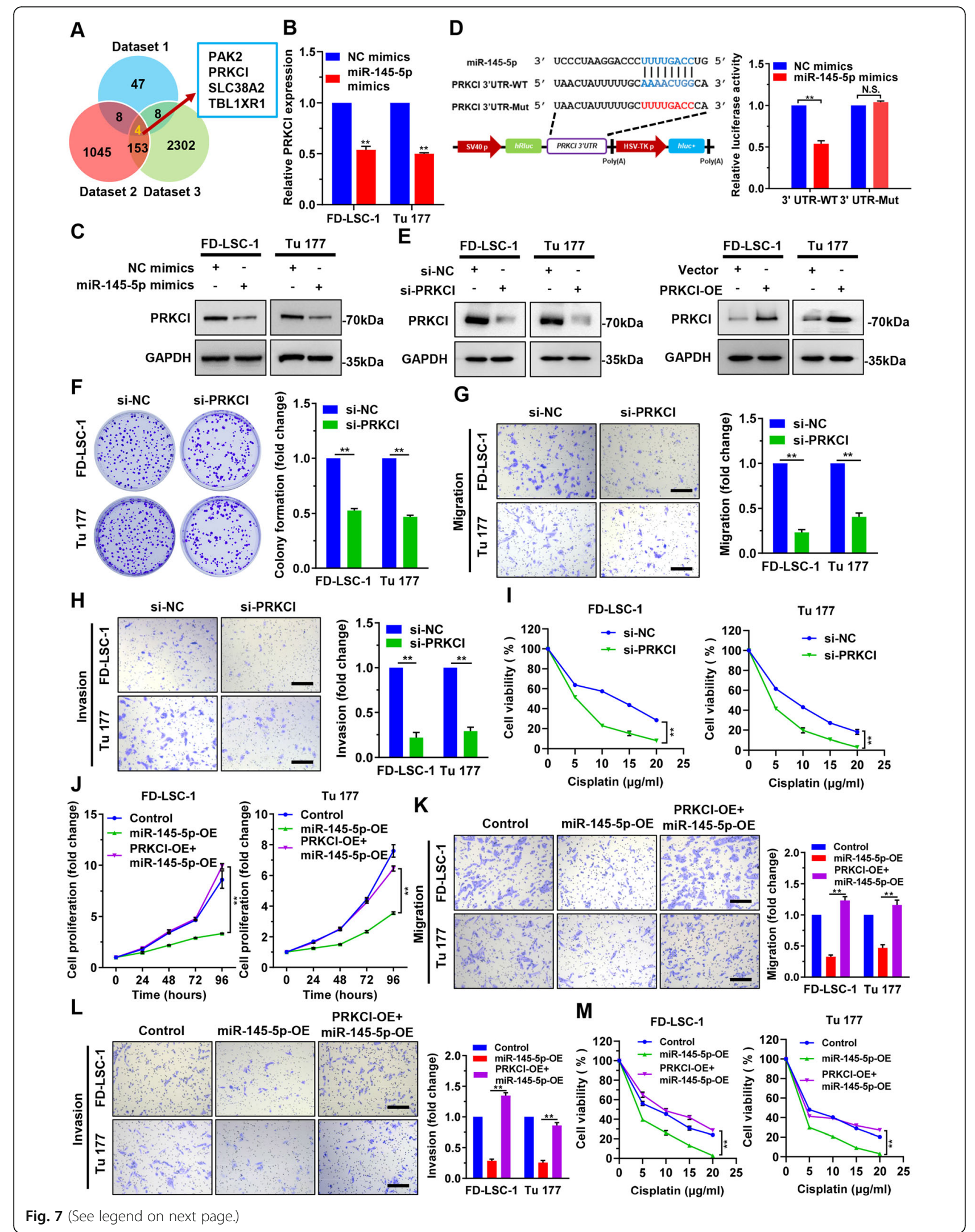




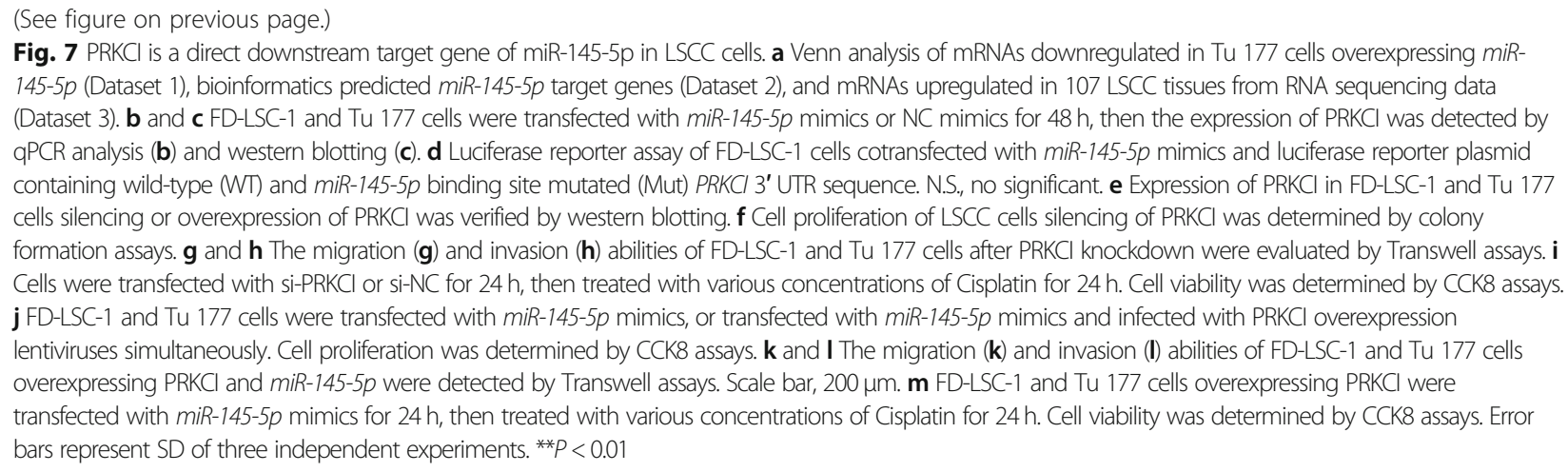

stages (Fig. 3c-e). Notably, the expression levels of circPARD3 increased progressively from $\mathrm{T} 1$ to $\mathrm{T} 4$ stage (Fig. 3c). Moreover, circPARD3 level was higher in lowly differentiated LSCC tissues than that in highly differentiated tissues (Fig. 3f). However, the $\operatorname{circPARD3}$ level was not significantly correlated with sex, age, smoking or alcohol consumption in LSCC patients $(p>0.05)$. We divided the LSCC tissues into the high-expression $(n=50)$ and lowexpression $(n=50)$ groups according to median circPARD3 expression level. Kaplan-Meier analysis showed that patients with high $\operatorname{circPARD} 3$ expression had much shorter survival (Fig. 3g). We also compared circPARD3 expression between LSCC cell lines (FD-LSC-1 and Tu 177) and normal control cell lines (HOK, MRC-5, and HEK293T). The results showed that circPARD3 expression was significantly higher in LSCC cells than control cells (Fig. 3h). Taken together, these data indicated that upregulation of circPARD3 is closely associated with malignant progression of LSCC, therefore $\operatorname{circPARD} 3$ may play an oncogenic role in LSCC.

\section{circPARD3 inhibits autophagy of LSCC cells}

To validate that circPARD3 regulates autophagy in LSCC, we transfected LSCC cells with circPARD3 overexpression plasmid and siRNAs, respectively. qPCR results demonstrated that circPARD3 expression in FDLSC-1 and Tu 177 cells transfected with circPARD3 overexpression plasmid and siRNAs was increased and decreased correspondingly (Fig. 4a and b), but linear PARD3 mRNA did not change significantly (Additional file 3: Figure S4). Detection of autophagy-related markers revealed that LC3-II level was decreased in FDLSC-1 and Tu 177 cells overexpressing $\operatorname{circPARD} 3$, but the level of the autophagy substrate protein p62 was increased (Fig. 4c). Conversely, circPARD3 knockdown led to increased LC3-II level but decreased p62 level (Fig. 4d). Next, we transfected mCherry-GFP-LC3B labeled LSCC cells with $\operatorname{circPARD} 3$ overexpression plasmid or siRNAs, and analyzed the effect of $\operatorname{circPARD} 3$ on autophagy flux by observing fluorescence changes. The numbers of green and red LC3 puncta were decreased in LSCC cells with circPARD3 overexpression (Fig. 4e). By contrast, the numbers of green and red LC3 puncta were increased after circPARD3 knockdown (Fig. 4f). These results confirmed that circPARD3 inhibits autophagy of LSCC cells.

To further clarify the relationship between autophagy and $\operatorname{circPARD} 3$ expression, LSCC cells were treated with the autophagy activator Rapamycin, or autophagy inhibitor 3-Methyladenine (3-MA). Results showed that circPARD3 expression did not significantly differ in FDLSC-1 and Tu 177 cells after activation or inhibition of autophagy (Fig. 4g). Collectively, circPARD3 acted as an upstream regulator to inhibit autophagy in LSCC cells, but autophagy level changes did not affect circPARD3 expression.

\section{circPARD3 promotes LSCC cell proliferation, migration,} invasion and chemoresistance by inhibiting autophagy CCK8 analysis showed that FD-LSC-1 and Tu 177 cell viability decreased after circPARD3 knockdown (Fig. 5a). Colony formation experiments exhibited that knockdown of circPARD3 decreased the clonogenic capacity of LSCC cells (Fig. 5b). Furthermore, knockdown of circPARD3 significantly reduced the migration and invasion abilities of FD-LSC-1 and Tu 177 cells (Fig. 5c and d), and increased the sensitivity of LSCC cells to the chemotherapeutic drug Cisplatin (Fig. 5e). These results demonstrated that circPARD3 acts as an oncogene to promote LSCC cell proliferation, migration, invasion and chemoresistance.

To determine whether circPARD3 affects the malignant phenotypes of LSCC cell by regulating autophagy, LSCC cells were transfected with si-circPARD3 and treated with the autophagy inhibitor 3-MA simultaneously. We found that 3-MA treatment reversed the effects of circPARD3 knockdown on FD-LSC-1 and Tu 177 cell proliferation, migration, invasion and chemosensitivity (Fig. 5f-i). Collectively, these data indicated that circPARD3 promotes 


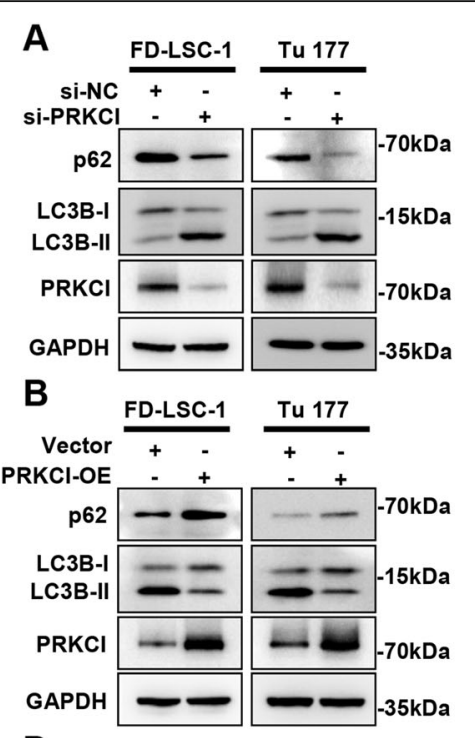

D
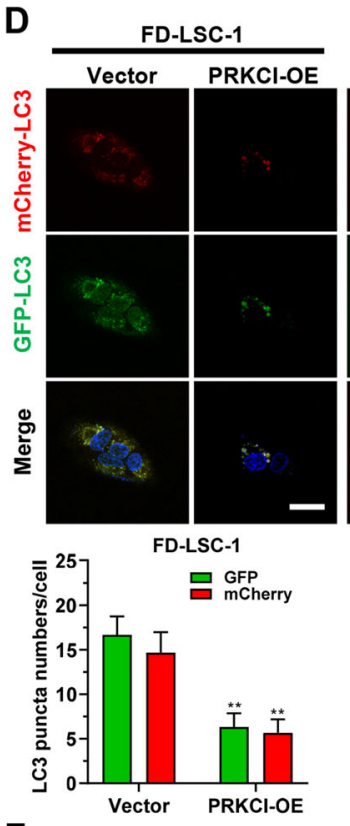

F

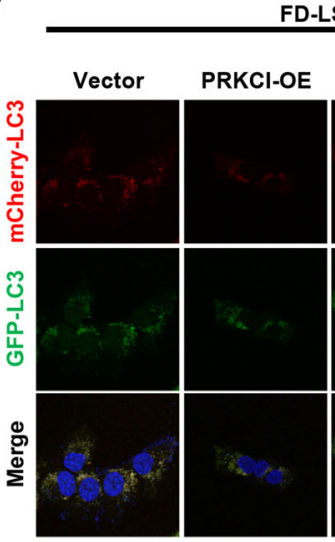

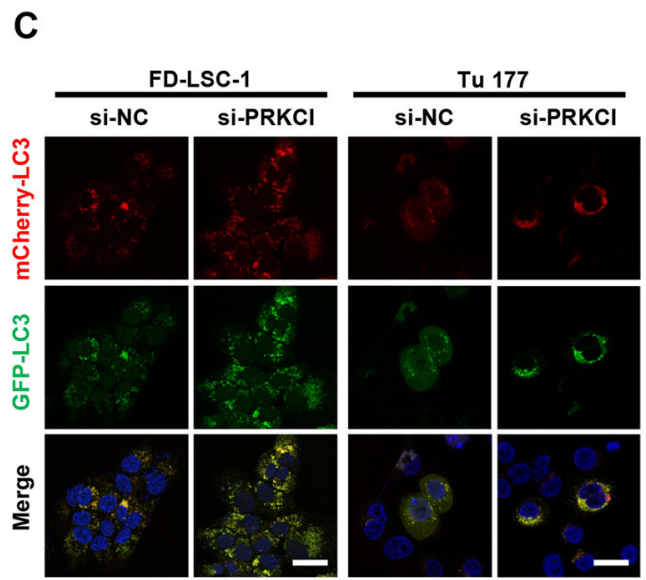

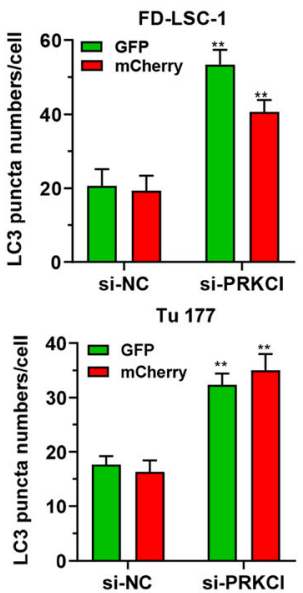

E
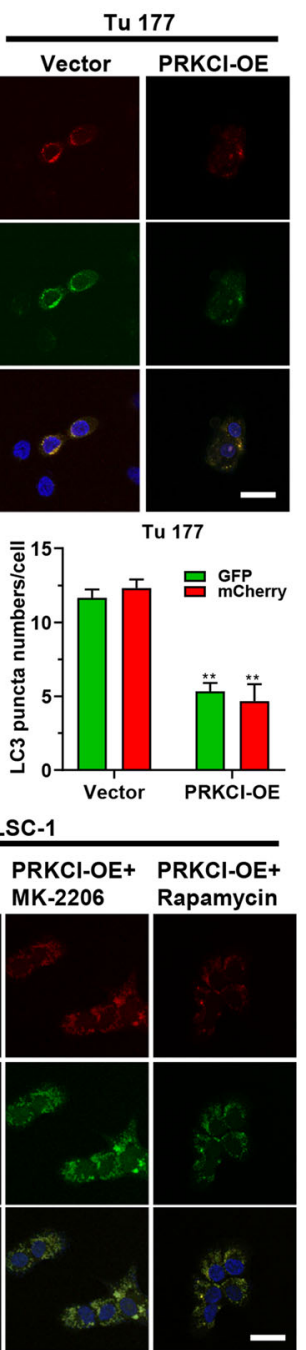

9
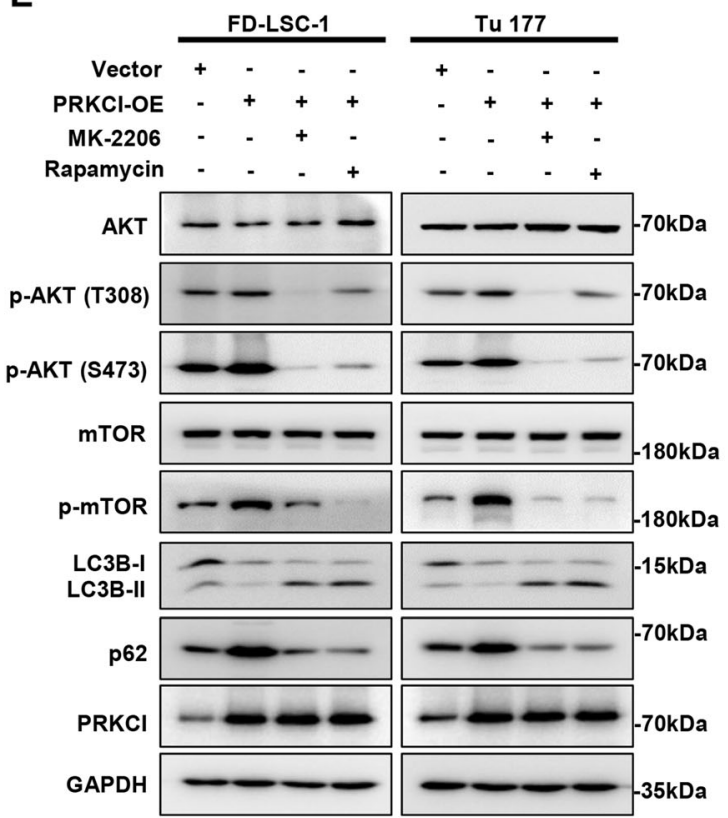

Tu 177

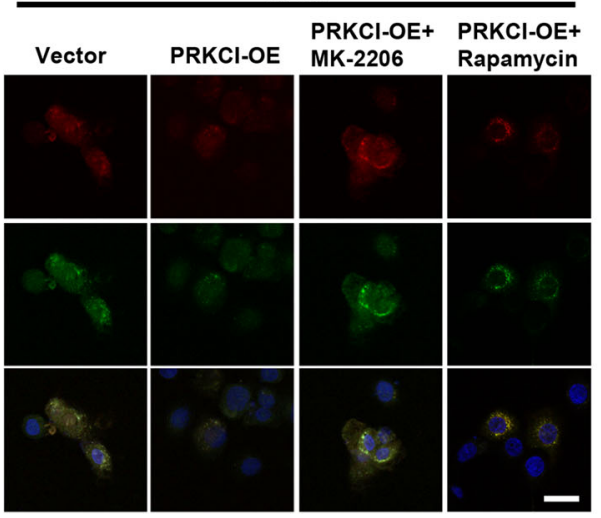

Fig. 8 (See legend on next page.) 
(See figure on previous page.)

Fig. 8 PRKCl inhibits LSCC cell autophagy via activating the Akt-mTOR axis. $\mathbf{a}$ and $\mathbf{b}$ The protein levels of PRKCI, LC3B, and p62 in FD-LSC-1 and Tu 177 cells with knockdown (a) or overexpression (b) of PRKCI were detected by Western blotting. $\mathbf{c}$ and $\mathbf{d}$ mCherry-EGFP-LC3B labeled FD-LSC1 and Tu 177 cells were transfected with si-PRKCI (c) or infected with PRKCl overexpression lentiviruses (d), autophagic flux was analyzed by confocal microscopy. e PRKCl overexpressing FD-LSC-1 and Tu 177 cells (PRKCI-OE) were treated with MK-2206 (10 $\mu$ M) or Rapamycin (100 nM) for $24 \mathrm{~h}$, the protein levels of phosphorylated Akt (p-AKT(T308), p-AKT(S473)), phosphorylated mTOR (p-mTOR), LC3B, and p62 were detected by western blotting. $\mathbf{f}$ mCherry-EGFP-LC3B labeled FD-LSC-1 and Tu 177 cells were infected with PRKCl overexpression lentiviruses for $48 \mathrm{~h}$, then treated with MK-2206 (10 $\mu \mathrm{M})$ or Rapamycin $(100 \mathrm{nM})$ for $24 \mathrm{~h}$. Autophagic flux was analyzed by confocal microscopy. Scale bar, $25 \mu \mathrm{m}$. Error bars represent SD of three independent experiments. ${ }^{* *} P<0.01$

LSCC cell proliferation, migration, invasion and chemoresistance by inhibiting autophagy.

\section{circPARD3 acts as a sponge of the tumor suppressor miR- 145-5p in LSCC}

The function of circRNA as miRNA sponge requires it to bind with AGO2 and miRNA to form a circRNAAGO2-miRNA complex [24]. RNA immunoprecipitation (RIP) experiments confirmed that circPARD3 bound to AGO2 (Fig. 6a), suggesting its role as a miRNA sponge. Bioinformatics analysis predicted that 54 miRNAs were potential targets of circPARD3 (Additional file 1: Table S10). Venn analysis of these 54 miRNAs and miRNAs downregulated in the 107 LSCC tissues obtained seven miRNA candidates, including miR-1298-5p, miR-136-5p, $m i R-139-5 p, m i R-145-5 p, m i R-338-3 p, m i R-100-5 p$, and $m i R-99 a-5 p$ (Fig. 6b). Next, Biotin-labeled probes that specifically target circPARD3 were used for RNA pulldown, then $\mathrm{qPCR}$ was used to detect these seven miRNAs. Results showed that $m i R-145-5 p$ was the highest enriched one among the 7 miRNAs (Fig. 6c). Furthermore, we found that only $m i R-145-5 p$ expression was significantly decreased after $\operatorname{circPARD} 3$ overexpression, whereas $\operatorname{circPARD} 3$ knockdown led to increased $m i R$ 145-5p expression in both FD-LSC-1 and Tu 177 cells (Fig. 6d; Additional file 3: Figure S5A and B). We constructed $\operatorname{circPARD} 3$ luciferase reporter plasmids with wild-type (circPARD3-WT) or mutant (circPARD3-Mut) $m i R-145-5 p$ binding sites. We observed that the luciferase activity was markedly decreased in cells cotransfected with $m i R-145-5 p$ mimics and circPARD3-WT, but not with circPARD3-Mut (Fig. 6e). These data suggested that $\operatorname{circPARD} 3$ serves as a sponge for $m i R$ 145-5p.

Next, we investigated whether $m i R-145-5 p$ affected autophagy levels in LSCC cells. Western blotting showed increased LC3-II level and decreased p62 level in FDLSC-1 and Tu 177 cells after transfection with miR-145$5 p$ mimics (Additional file 3: Figure S6A). Conversely, LC3-II was decreased, and p62 was increased after miR145-5p inhibitor transfection (Additional file 3: Figure S6B). Autophagic flux analysis showed the numbers of green and red LC3 puncta increased in the cells transfected with miR-145-5p mimics but decreased in those transfected with $m i R-145-5 p$ inhibitor (Additional file 3: Figure S6C), indicating that $m i R-145-5 p$ enhances the autophagy levels in LSCC cells.

Since $m i R-145-5 p$ acts as a tumor suppressor to inhibit LSCC cell proliferation, migration and invasion [25], we wonder whether circPARD3 inhibits autophagy and promotes malignant progression of LSCC by antagonizing $m i R-145-5 p$. Western blotting and autophagy flux analysis showed that overexpression of $\operatorname{circPARD} 3$ reversed the activation effect of the miR-145-5p mimics on autophagy (Fig. 6f; Additional file 3: Figure S7). CCK8 and colony formation assays revealed that overexpression of cir$c P A R D 3$ reversed the inhibitory effect of $m i R-145-5 p$ mimics on cell proliferation (Fig. $6 g$ and $h$ ). Transwell analysis showed that overexpression of $\operatorname{circPARD} 3$ rescued the decreased migration and invasion caused by $m i R-145-$ $5 p$ mimics transfection (Fig. $6 \mathrm{i}$ and $\mathrm{j}$ ). Moreover, cir$c P A R D 3$ overexpression also antagonized the chemosensitization effect of $m i R-145-5 p$ mimics on FD-LSC-1 and $\mathrm{Tu} 177$ cells (Fig. 6k). These data suggested that cirCPARD3 inhibits autophagy by sponging the tumor suppressor $m i R-145-5 p$, thereby promoting LSCC cell proliferation, migration, invasion, and chemoresistance.

\section{$\mathrm{PRKCl}$ is a direct target gene of miR-145-5p, which functions as a driver gene in LSCC}

To explore the direct downstream target gene of $m i R-145-5 p$, we investigated mRNAs that might be regulated by $m i R-145-5 p$ in LSCC cells via microarray analysis. Sixty-seven mRNAs were downregulated in Tu 177 cells with miR-145-5p overexpression (Dataset 1, Additional file 1: Table S11). Bioinformatics analysis prediction of $m i R-145-5 p$ target gene obtained 1210 potential target genes (Dataset 2, Additional file 1: Table S12). Venn analysis of Dataset 1, Dataset 2, and mRNAs upregulated in the 107 LSCC tissues compared to their paired ANM tissues from the RNA sequencing data (Dataset 3, Additional file 1: Table S13) obtained four intersection genes, $P A K 2, P R K C I$, SLC38A2 and TBL1XR1 (Fig. 7a). Furthermore, we found that only PRKCI mRNA and protein levels were significantly decreased in LSCC cells transfected with $m i R-145-5 p$ mimics (Fig. $7 \mathrm{~b}$ and c; Additional file 3: Figure S8). Luciferase reporter assays showed 
A

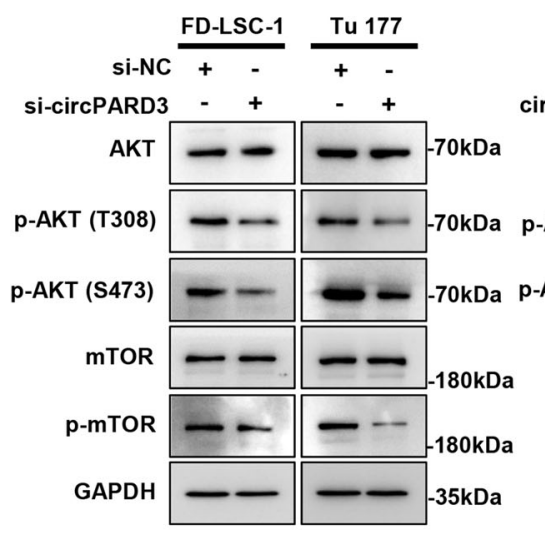

C
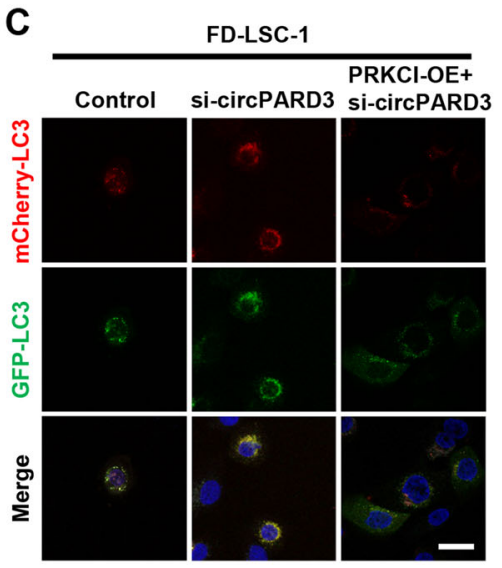

D
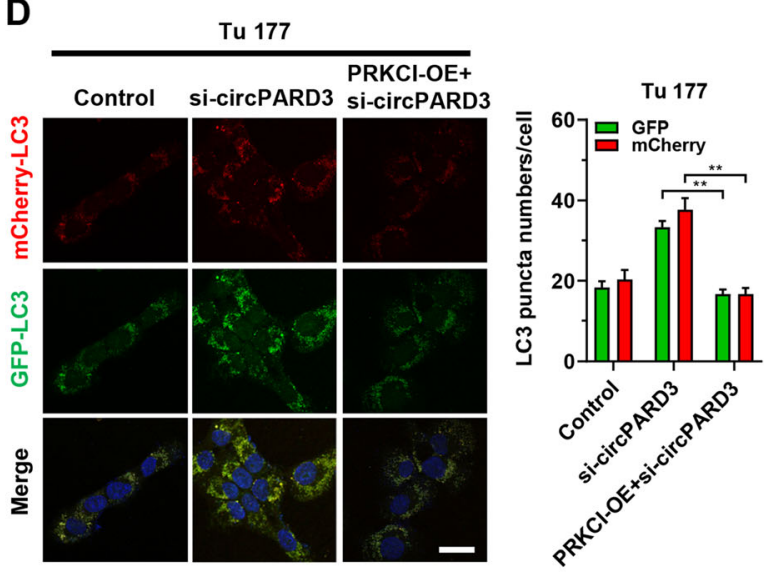

H
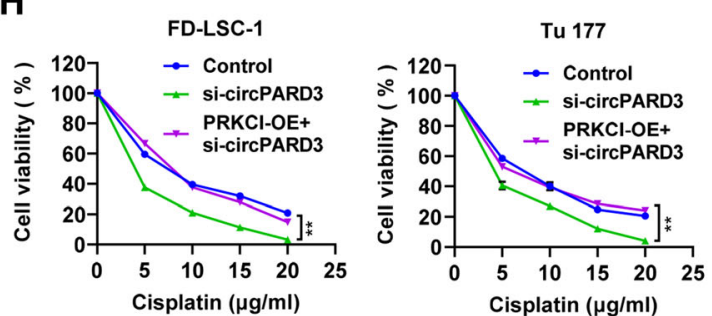

B

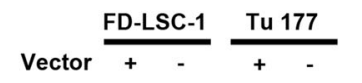

E
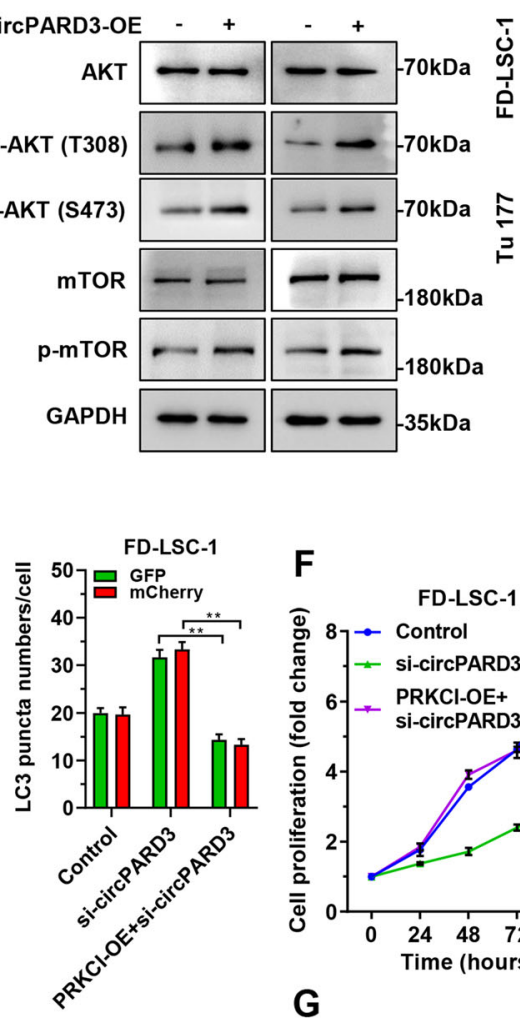

$\mathbf{F}$
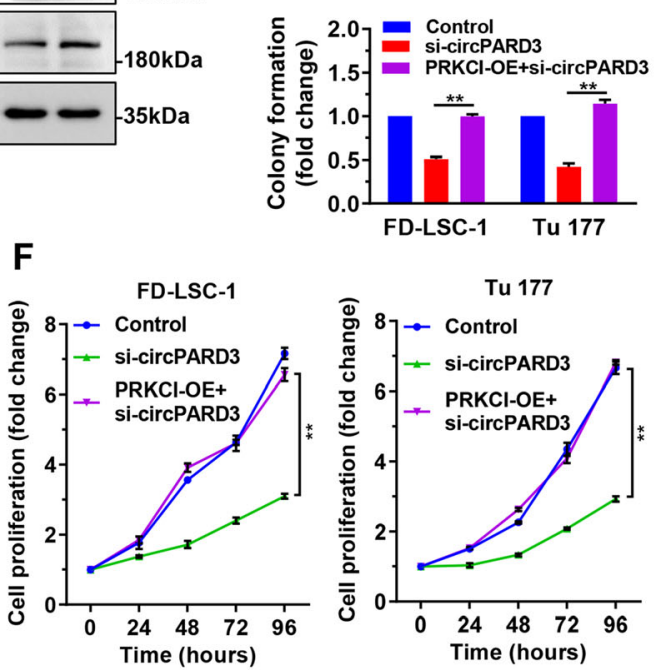

PRKCl-OE+

G

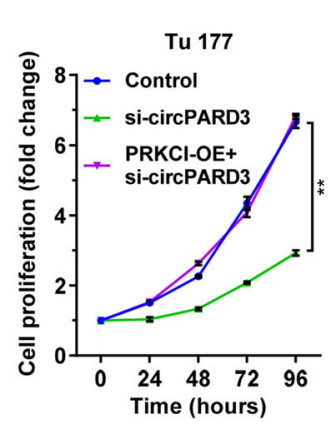

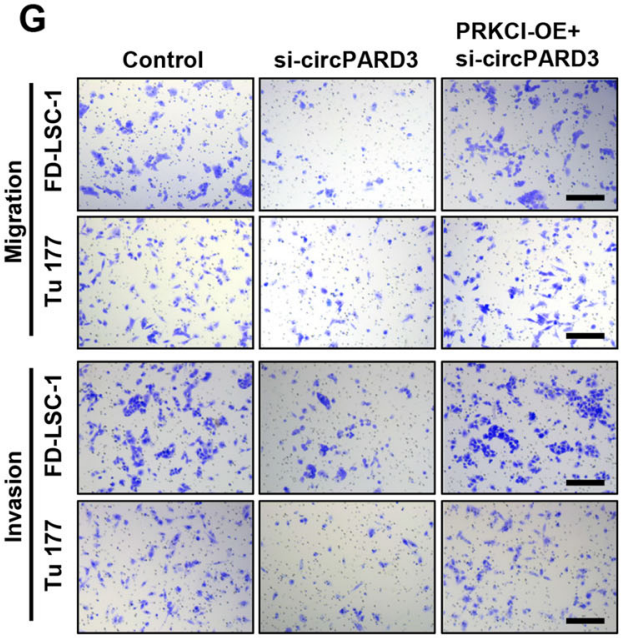

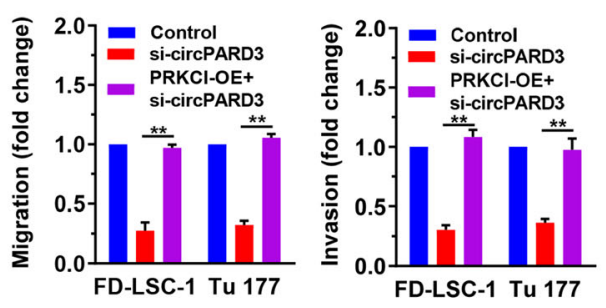

Fig. 9 (See legend on next page.) 


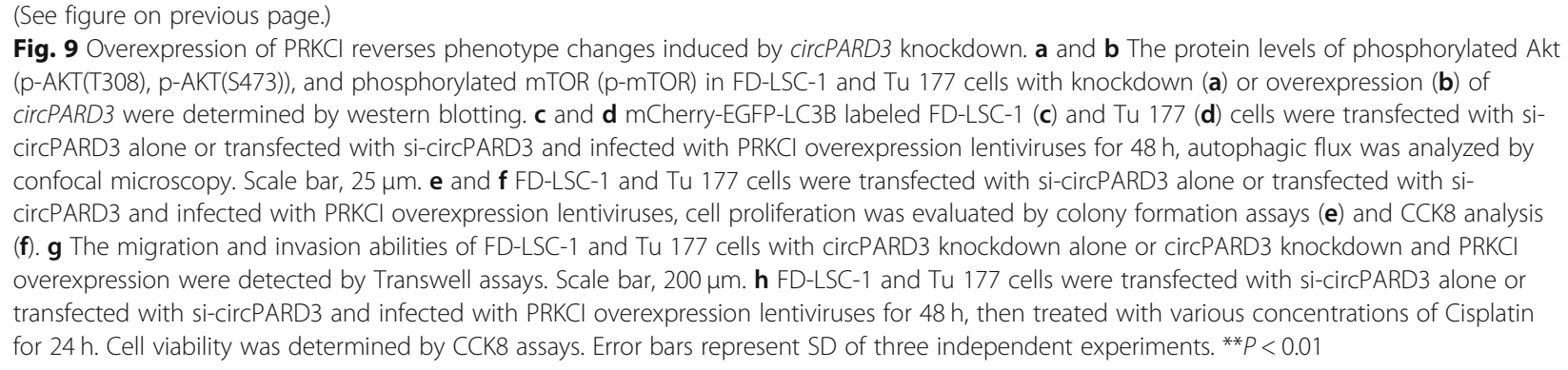

that cotransfection of $m i R-145-5 p$ mimics reduced the luciferase activity of the wild-type PRKCI 3' UTR reporter, but not the one with $m i R-145-5 p$ binding site mutated (Fig. $7 \mathrm{~d}$ ). These data suggested that PRKCI is a direct target gene of miR-145-5p.

We further tested the effect of PRKCI on LSCC cell proliferation, migration, invasion and chemosensitivity. Transfection of LSCC cells with siRNAs targeting PRKCI (si-PRKCI) or infection with PRKCI overexpression lentiviruses (PRKCI-OE) changed PRKCI levels successfully (Fig. 7e). Colony formation and Transwell assays indicated that knockdown of PRKCI inhibited LSCC cell proliferation, migration and invasion (Fig. 7f-h), while overexpression of PRKCI promoted LSCC cell proliferation, migration and invasion (Additional file 3: Figure S9A-C). Furthermore, knockdown of PRKCI enhanced, but overexpression of PRKCI decreased LSCC sensitivity to chemotherapeutic drug Cisplatin (Fig. 7i; Additional file 3: Figure S9D). Rescue experiments demonstrated that PRKCI overexpression reversed the inhibitory effect of $m i R-145-5 p$ on LSCC cell proliferation, migration, and invasion (Fig. 7j-1), and reduced the sensitivity of cells to Cisplatin (Fig. $7 \mathrm{~m}$ ). These results indicated that $P R K C I$, as a direct downstream target gene of miR-145$5 p$, functions as a driver gene in LSCC to promote cell proliferation, migration, invasion and chemoresistance.

\section{PRKCl inhibits autophagy by activating the Akt-mTOR signaling axis}

Next, we investigated the effect of PRKCI on autophagy in LSCC cells. FD-LSC-1 and Tu 177 cells with PRKCI knockdown showed higher LC3-II and lower p62 levels than that of control cells (Fig. 8a). By contrast, LC3-II level was decreased, but p62 level was increased after PRKCI overexpression (Fig. 8b). Autophagy flux detection revealed that autophagy level was increased in LSCC cells with PRKCI knockdown and decreased in cells with PRKCI overexpression (Fig. 8c and d), suggesting that PRKCI is an autophagy suppressor in LSCC.

As a member of the protein kinase C family, PRKCI directly phosphorylates Akt and activates the Akt signaling pathway, and the activated Akt pathway could suppress autophagy by phosphorylating mTOR [26]. To examine whether PRKCI inhibits autophagy in LSCC cells by activating the Akt-mTOR signaling axis, PRKCI overexpressing LSCC cells were treated with Akt phosphorylation inhibitor MK-2206 or mTOR phosphorylation inhibitor Rapamycin. We observed that PRKCI overexpression enhances protein phosphorylation levels of Akt and mTOR (Fig. 8e). However, MK-2206 and Rapamycin treatment eliminated the increased phosphorylation of Akt and mTOR induced by PRKCI (Fig. 8e). Furthermore, we found that MK-2206 and Rapamycin treatment reversed the inhibitory effect of PRKCI on FD-LSC-1 and Tu 177 cell autophagy (Fig. 8e and f). These data confirmed that PRKCI inhibits LSCC cell autophagy by activating the Akt-mTOR signaling axis.

circPARD3 inhibits LSCC cell autophagy via the PRKCl-AktmTOR pathway

Subsequently, we further assessed the effects of circPARD3 on the PRKCI-Akt-mTOR pathway. Western blotting revealed that circPARD3 knockdown decreased protein phosphorylation levels of Akt and mTOR (Fig. 9a), while circPARD3 overexpression led to increased phosphorylation of Akt and mTOR (Fig. 9b), suggesting that circPARD3 is able to activate the PRKCIAkt-mTOR pathway. Autophagy flux analysis showed that PRKCI overexpression attenuated the increased autophagy induced by circPARD3 knockdown (Fig. 9c and d). Furthermore, we found that PRKCI overexpression rescued the decreased cell proliferation, migration, invasion and chemoresistance abilities caused by $\operatorname{circPARD} 3$ knockdown (Fig. 9e-h).

To validate the significance of the PRKCI-Akt-mTOR pathway for circPARD3-mediated autophagy inhibition, cirCPARD3 overexpressing LSCC cells were treated with MK2206 or Rapamycin, respectively. Western blotting and autophagy flux analysis showed that inhibition of Akt or mTOR phosphorylation reactivated autophagy inhibited by cirCPARD3 overexpression (Fig. 10a-c). We further compared the changes of autophagy levels in circPARD3 knockdown cells treated with the Akt activator SC79 or the mTOR activator MHY1485. Expectedly, autophagy levels in SC79 or MHY1485 treatment cells were lower than that of nontreatment controls (Fig. 10d and e; Additional file 3: Figure S10). 

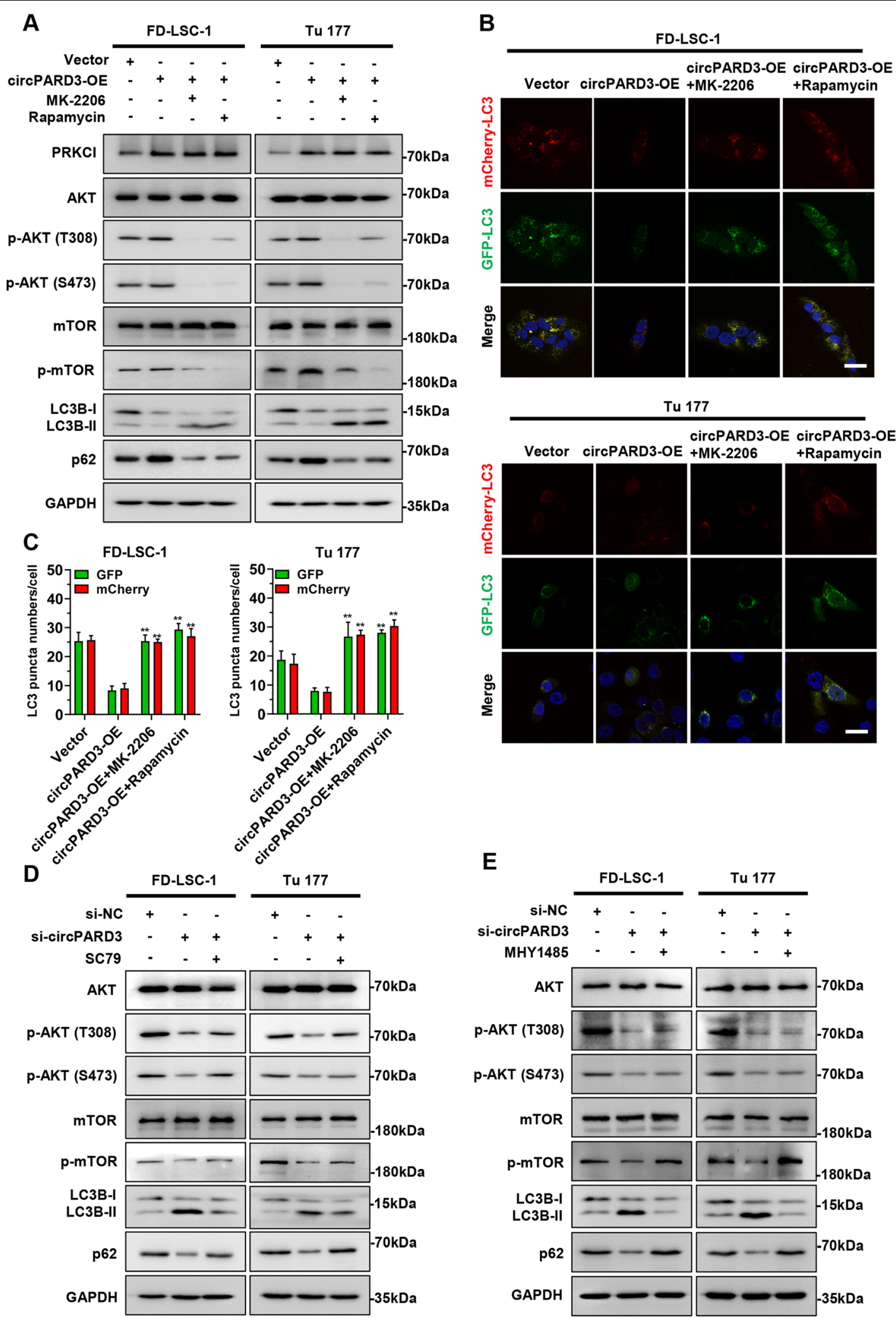

Fig. 10 (See legend on next page.) 
(See figure on previous page.)

Fig. 10 cirCPARD3 inhibits autophagy of LSCC cells via the PRKCI-Akt-mTOR pathway. a FD-LSC-1 and Tu 177 cells with circPARD3 overexpression (circPARD3-OE) were treated with MK-2206 $(10 \mu \mathrm{M})$ or Rapamycin $(100 \mathrm{nM})$ for $24 \mathrm{~h}$, then the protein levels of phosphorylated Akt (p-AKT(T308), p-AKT(S473)), phosphorylated mTOR (p-mTOR), LC3B, and p62 were detected by western blotting. $\mathbf{b}$ and $\mathbf{c}$ mCherry-EGFP-LC3B labeled FD-LSC-1 and Tu 177 cells were infected with circPARD3 overexpression lentiviruses for $48 \mathrm{~h}$, then treated with MK-2206 (10 $\mu$ M) or Rapamycin (100 nM) for $24 \mathrm{~h}$. Autophagic flux was analyzed by confocal microscopy. Representative confocal microscopy images (b) and quantitative data (c) were shown. Scale bar, $25 \mu \mathrm{m}$. $\mathbf{d}$ and e FD-LSC-1 and Tu 177 cells were transfected with si-circPARD3 for $24 \mathrm{~h}$, then cells were treated with Akt activator SC79 $(5 \mu \mathrm{g} / \mathrm{ml})(\mathbf{d})$ or mTOR activator MHY1485 (10 $\mu \mathrm{M})(\mathbf{e})$ for $24 \mathrm{~h}$. The protein levels of phosphorylated Akt (p-AKT(T308), p-AKT(S473)), phosphorylated mTOR (p-mTOR), LC3B, and p62 were detected by western blotting. Error bars represent SD of three independent experiments. ${ }^{* *} P<0.01$

Collectively, these results suggested that the PRKCI-AktmTOR signaling pathway is the main pathway mediating the inhibitory effect of circPARD3 on autophagy in LSCC cells.

\section{Silencing of circPARD3 inhibits tumorigenicity and enhances chemosensitivity of LSCC cell in preclinical models}

To further investigate the functional role of $\operatorname{circPARD} 3$ in LSCC in vivo, we constructed FD-LSC-1 cells with stably knockdown of circPARD3 (sh-circPARD3) and control cells (sh-NC), and established xenograft tumor models in BALB/c nude mice. The tumor-bearing mice were treated with Cisplatin or normal saline for 3 weeks (the experimental group was intraperitoneally injected with $1 \mathrm{mg} / \mathrm{kg}$ of Cisplatin every 2 days; the control group was injected with the equal volume of normal saline). Compared with the control group, xenograft tumor growth rate was slower, and tumor weight was significantly lower in the sh-circPARD3 group (Fig. 11a and b). Notably, the sh-circPARD3 group treated with Cisplatin showed the slowest growth rate among all four groups (Fig. 11a and b). qPCR analysis demonstrated that $\operatorname{circPARD} 3$ was decreased, but miR-145-5p was increased in the sh-circPARD3 groups when compared with the sh-NC groups (Fig. 11c). Hematoxylin and eosin staining showed that the tumor cells in shcircPARD3 knockdown group of xenograft tumors was significantly reduced (Fig. 11d).

IHC staining revealed that the expression levels of PRKCI, phosphorylated Akt, phosphorylated mTOR, and p62 were decreased obviously in the tumors collected from sh-circPARD3 groups compared with those from the sh-NC groups (Fig. 11e). Immunofluorescence showed increased LC3 dots in xenograft tumors collected from shcircPARD3 groups (Fig. 11e). Furthermore, the proliferation marker Ki-67 and the EMT markers N-cadherin, and Vimentin were decreased in xenograft tumors with cirCPARD3 knockdown (Fig. 11f). In contrast, E-cadherin level was increased in xenograft tumors with circPARD3 knockdown (Fig. 11f). These results demonstrated that silencing of circPARD3 inhibited tumorigenicity and enhanced chemosensitivity of LSCC cells in vivo by enhancing autophagy through the PRKCI-Akt-mTOR pathway.

\section{Discussion}

LSCC is characterized by local recurrences, lymph node metastasis and poor sensitivity to chemotherapeutic drugs, which severely restricts the effects of LSCC treatment $[2,27]$. Autophagy plays critical roles in regulation of invasion, metastasis, and chemosensitivity in various type of cancers [28-32]. Therefore, scientists and clinicians are attempt to explore new cancer treatment approach by modulating autophagy. In this study, we found that the autophagy substrate protein p62 was significantly upregulated in LSCC tissues, and high level of p62 was associated with malignant progression and poor prognosis of LSCC patients, indicating that autophagy inhibition is an important molecular event in LSCC initiation and development. Moreover, these data also suggested that the anti-tumor effect of autophagy is continuously inhibited during LSCC initiation and progression. Therefore, reactivation of autophagy may be able to suppress LSCC cell malignant behaviors. Remarkably, our data demonstrated that upregulation of autophagy level inhibited proliferation, migration, invasion, and enhanced chemosensitivity of LSCC cells to Cisplatin in vitro and in vivo. Similarly, Zhu et al. reported that upregulation of autophagy level inhibited nasopharyngeal carcinoma cell invasion and metastasis [33], confirming that activation of autophagy might be a new strategy for LSCC treatment.

We further screened the autophagy-related circRNAs by RNA sequencing of LSCC tissues and autophagic flux analysis, and identified a novel autophagy-suppressive circRNA $\operatorname{circPARD3}$. $\operatorname{circPARD} 3$ was abnormally upregulated in LSCC tissues compared with their paired normal tissues. Importantly, we found that high circPARD3 level was closely associated with malignant progression and poor prognosis of LSCC patients. In vivo and in vitro experiments confirmed that circPARD3 promoted LSCC cell proliferation, migration, invasion and chemoresistance by inhibiting autophagy. To the best of our knowledge, for the first time, this study reveals the intrinsic molecular mechanism of LSCC maintains low autophagy levels via circRNA and clarifies the oncogenic role of circPARD3 in LSCC. Our findings suggested that cirCPARD3 can serve as a biomarker for diagnosis and prognosis of LSCC. More importantly, circPARD3 can 
A

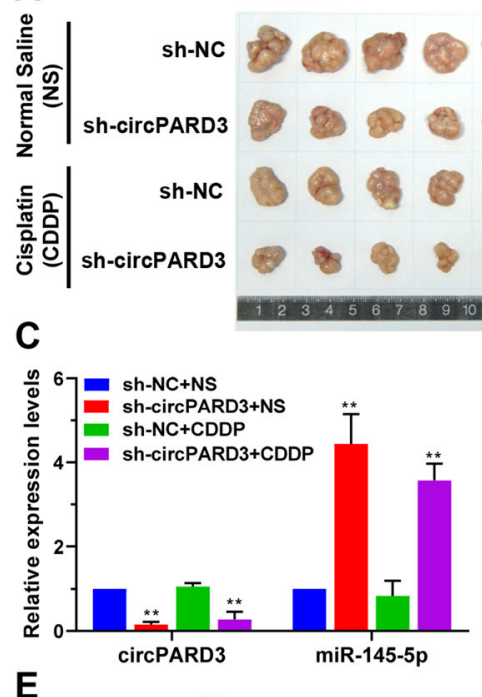

E

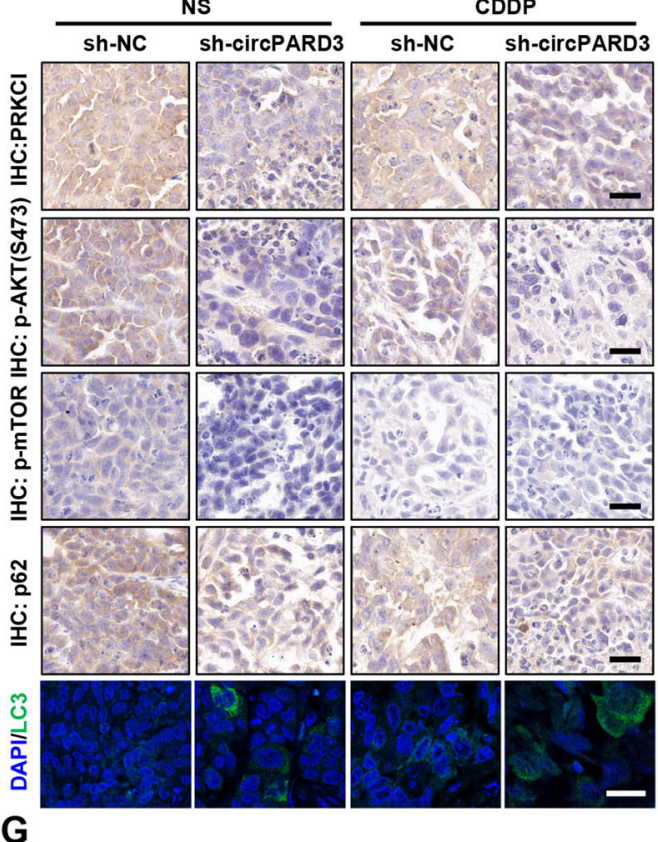

B
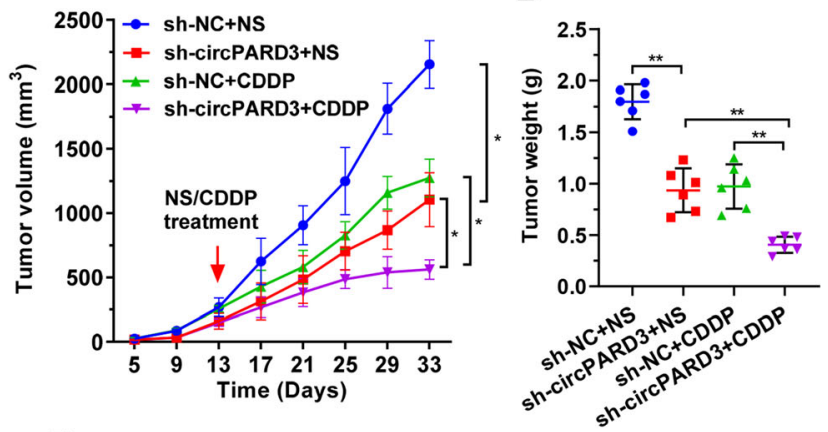

D

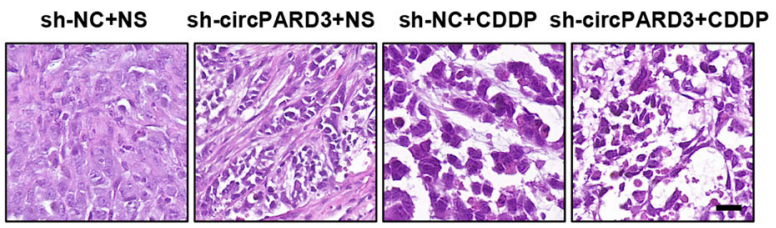

Tumor cell

(1)

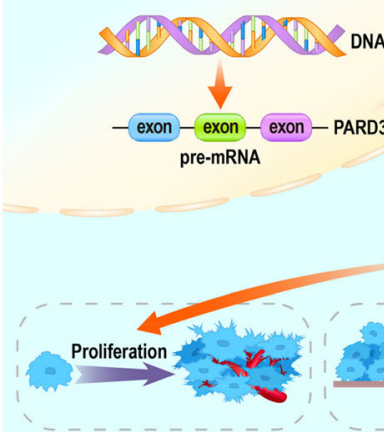

NUGLEUS

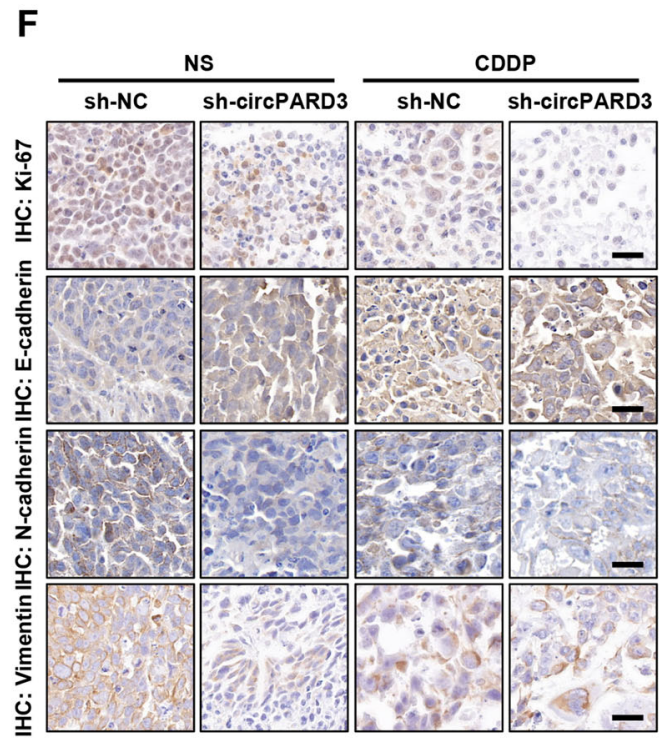

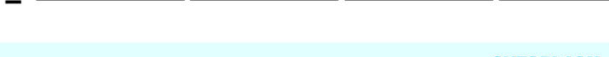

Fig. 11 (See legend on next page.) 
(See figure on previous page.)

Fig. 11 Silencing of cirCPARD3 suppresses tumorigenicity and enhances chemosensitivity of LSCC cells in vivo. a Effects of circPARD3 on LSCC xenograft tumor growth and chemosensitivity in vivo. Left: Representative images of tumors in nude mice after subcutaneous injection of FDLSC-1 cells with circPARD3 knockdown (sh-circPARD3) and treated with Cisplatin $(n=6)$. Right: Tumor growth curve was plotted using xenograft tumor volume data. $\mathbf{b}$ Tumor weight was measured after tumor excision. $\mathbf{c}$ The relative expression levels of circPARD3 and miR-145-5p in xenograft tumors were determined by qPCR analysis. $\mathbf{d}$ Hematoxylin and eosin staining revealed the structure of xenograft tumors. Scale bar, $20 \mu \mathrm{m}$. e IHC staining of PRKCl, p-AKT(S473), p-mTOR, p62 and immunofluorescence of LC3B in xenograft tumors. Scale bar, $25 \mu \mathrm{m}$. $\mathbf{f} \| \mathrm{HC}$ staining of Ki-67, E-cadherin, N-cadherin, and Vimentin in xenograft tumors. Scale bar, $25 \mu \mathrm{m}$. g Schematic illustration indicates the mechanism by which circPARD3 inhibits autophagy to promote malignant progression and chemoresistance of LSCC via activating the PRKCI-Akt-mTOR pathway. NS, Normal Saline. Data are mean \pm SD. ${ }^{*} P<0.05,{ }^{* *} P<0.01$

serve as a new biomarker and target for autophagy detection and modulation, which might be used for controlling autophagy levels in different developmental stages of LSCC.

Sponging target miRNAs and inhibiting their functions are classic mechanisms of circRNA. circRNA-002178 upregulates Programmed death-ligand 1 (PD-L1) expression in lung adenocarcinoma by sponging miR-34 [34]. circAMOTL1 enhances expression of the parent gene AMOTL1 by sponging miR-485-5p, which promotes cervical cancer progression [35]. Furthermore, $\operatorname{circCDYL}$ upregulates ATG7 and ULK1 expression by sponging miR-1275, thereby enhancing the autophagy levels and malignant progression of breast cancer [36]. We found that $\operatorname{circPARD} 3$ was mainly localized in the cytoplasm and inhibited LSCC cell autophagy by sponging miR-145-5p . Previous studies reported that miR-145-5p promotes autophagy of cardiomyocytes and human umbilical cordderived mesenchymal stem cells by inhibiting the Akt signaling pathway [37, 38], supporting our conclusion that miR-145-5p promotes autophagy in LSCC cells.

As a member of the protein kinase C family, PRKCI affects tumorigenesis, progression and chemosensitivity by regulating the Wnt/beta-catenin pathway and immune microenvironment in lung cancer, ovarian cancer and alveolar rhabdomyosarcoma [39-41]. Therefore, PRKCI is considered an important target for cancer treatment. Our RNA sequencing data showed that PRKCI was upregulated in LSCC, and we demonstrated that PRKCI was a new target gene of miR-145-5p. Moreover, functional studies demonstrated that PRKCI inhibited autophagy and promoted LSCC cell proliferation, migration, invasion and chemoresistance. $\mathrm{Qu}$ et al. found that knockdown of PRKCI in U2OS cells resulted in elevated autophagy levels and inhibiting the malignant cell phenotype [26]. Consistently, our study revealed that PRKCI enhanced Akt and mTOR phosphorylation and inhibited LSCC cell autophagy. Furthermore, blocking Akt and mTOR activity using small-molecule inhibitors eliminated the inhibitory effect of PRKCI on autophagy, indicating that PRKCI inhibits autophagy by activating the Akt-mTOR pathway in LSCC cells. In addition, our data demonstrated that circPARD3 inhibited autophagy and promoted malignant progression and chemoresistance of LSCC cells via activating the
PRKCI-Akt-mTOR pathway. Taken together, our study linked circPARD3 with autophagy, LSCC progression and chemoresistance.

Finally, our study revealed that knockdown of cirCPARD3 inhibited the tumorigenicity and enhanced chemosensitivity of LSCC cells in preclinical models. Furthermore, IHC staining results confirmed that downregulation of $\operatorname{circPARD} 3$ inhibited the PRKCI-AktmTOR pathway and activated autophagy in vivo. These results indicated that activating autophagy is a potential strategy for LSCC treatment, and developing techniques for circPARD3 detection and intervention to restore autophagy activity have great potential for LSCC diagnosis and treatment.

\section{Conclusion}

In summary, we demonstrated that autophagy is inhibited in LSCC, and that circPARD3 inhibits autophagy to promote malignant and chemoresistance of LSCC. Our study further revealed the mechanism of circPARD3 regulates autophagy via the miR-145-5p-PRKCI-Akt-mTOR pathway (Fig. 11g). Collectively, these findings provide new insights into the role and mechanism of circRNAs in autophagy, progression, and chemoresistance of LSCC. Moreover, the current study highlights the importance of restoring autophagy in LSCC treatment, and demonstrates the potential of $\operatorname{circPARD} 3$ as a biomarker and effective target for LSCC diagnosis and treatment.

\section{Supplementary Information}

The online version contains supplementary material available at https://doi. org/10.1186/s12943-020-01279-2.

Additional file 1: Table S1. Clinicopathological characteristics of 138 LSCC samples (cohort 1) for IHC staining of p62. Table S2.

Clinicopathological characteristics of 107 LSCC samples (cohort 2) for RNA sequencing. Table S3. Clinicopathological characteristics of 100 LSCC samples (cohort 3) for qPCR analysis of circPARD3. Table S4. Detailed information of inhibitors. Table S5. Cell lines and growth medium. Table S6. Primer information for qPCR analysis. Table S7. Differentially expressed circRNAs in RNA sequencing data of $107 \mathrm{LSCC}$ and paired ANM tissues. Table S8. Differentially expressed miRNAs in RNA sequencing data of 107 LSCC and paired ANM tissues. Table S9. Differentially expressed mRNAs in RNA sequencing data of 107 LSCC and paired ANM tissues. Table S10. Prediction of circPARD3 binding miRNAs by Circlnteractome. Table S11. Downregulated genes in Tu 177 cells transfected with miR-145-5p mimics analyzed by microarray. Table S12. 
Prediction of miR-145-5p target gene by Targetminer. Table S13. Upregulated mRNAs in RNA sequencing data of 107 LSCC and paired ANM tissues

Additional file 2. Supplemental Materials and Methods.

Additional file 3: Figure S1. The flow chart for screening and verifying autophagy suppressive cirCPARD3 in LSCC. Figure S2. FD-LSC-1 and Tu 177 cells were transfected with Cy3 labeled si-NC (si-NC-Cy3), NC mimics (NC mimics-Cy3), or NC inhibitor (NC inhibitor-Cy3) for $48 \mathrm{~h}$. Nuclei were stained with DAPI (blue). Transfection efficiency was evaluated by imaging with confocal microscopy. Red dot represents siRNA, miRNA mimics, or miRNA inhibitor. Scale bar, 50 m. Figure $\mathbf{S 3}$. Verification of the structure features of circPARD3. a Expression of circPARD3 in FD-LSC-1 and Tu 177 cells was verified by RT-PCR. Agarose gel electrophoresis showed that divergent primers amplified circPARD3 in CDNA but not genomic DNA (gDNA). GAPDH served as a negative control. b Stability of cirCPARD3 and linear PARD3 mRNA was assessed by RNase $R$ treatment and RT-PCR analysis. Figure S4. FD-LSC-1 and Tu 177 cells were infected with circPARD3 overexpression lentiviruses (circPARD3-OE) or transfected with si-circPARD3 (si-circ-1, si-circ-2) for $48 \mathrm{~h}$. Expression level of linear PARD3 mRNA was determined by qPCR analysis. Error bars represent SD of three independent experiments. N.S., no significant. Figure S5. Expression levels of potential circPARD3 target miRNAs in FD-LSC-1 and Tu 177 cells with overexpression (a) or knockdown (b) of circPARD3 were determined by $\mathrm{qPCR}$ analysis. Error bars represent SD of three independent experiments. ${ }^{*} P<0.05,{ }^{*} P<0.01$. Figure S6. The effects of miR-145-5p on LSCC cell autophagy. a and b FD-LSC-1 and Tu 177 cells were transfected with miR-145-5p mimics (a) or inhibitor (b) for $48 \mathrm{~h}$. Expression levels of p62 and LC3B were detected by western blotting. c FD-LSC-1 and Tu 177 cells were transfected with miR-145-5p mimics or inhibitor for $48 \mathrm{~h}$. Autophagic flux was analyzed by confocal microscopy. Representative images (Top) and statistical data (Bottom) were shown. Scale bar, $25 \mu \mathrm{m}$. Error bars represent SD of three independent experiments. ${ }^{*} P<0.05$, ${ }^{* *} P<0.01$. Figure S7. $m$ Cherry-EGFP-LC3B labeled FD-LSC-1 and Tu 177 cells were transfected with miR-145-5p mimics alone or infected with cirCPARD3 overexpression lentiviruses simultaneously for $48 \mathrm{~h}$. Autophagic flux was analyzed by confocal microscopy. Representative images (Top) and statistical data (Bottom) were shown. Scale bar, $25 \mu \mathrm{m}$. Error bars represent SD of three independent experiments. ${ }^{*} P<0.05,{ }^{* *} P<0.01$. Figure S8. FD-LSC-1 and Tu 177 cells were transfected with miR-145-5p mimics or NC mimics for $48 \mathrm{~h}$, then expression levels of PAK2, SLC38A2, and TBL1XR1 were determined by QPCR analysis. Error bars represent SD of three independent experiments. N.S., no significant. Figure S9. The effects of PRKCl overexpression on LSCC cell proliferation, migration, invasion, and chemoresistance. a Cell proliferation of FD-LSC-1 and Tu 177 cells overexpressing PRKCI was determined by colony formation assays. $\mathbf{b}$ and $\mathbf{c}$ The migration (b) and invasion (c) abilities of FD-LSC-1 and Tu 177 cells overexpressing PRKCI were evaluated by Transwell assays. Scale bar, $200 \mu \mathrm{m}$. d FD-LSC-1 and Tu 177 cells overexpressing PRKCI were treated with various concentrations of Cisplatin for $24 \mathrm{~h}$. Cell viability was determined by CCK8 assays. Error bars represent SD of three independent experiments. ${ }^{*} P<0.01$. Figure S10. FD-LSC-1 and Tu 177 cells were transfected with si-circPARD3 for $24 \mathrm{~h}$, then treated with Akt activator SC79 $(5 \mu \mathrm{g} / \mathrm{ml})$ or mTOR activator MHY1485 $(10 \mu \mathrm{M})$ for $24 \mathrm{~h}$. Autophagic flux was analyzed by confocal microscopy. Representative images (Left) and statistical data (Right) were shown. Scale bar, $25 \mu \mathrm{m}$. Error bars represent SD of three independent experiments. ${ }^{* *} P<0.01$.

\section{Abbreviations}

LSCC: Laryngeal squamous cell carcinoma; ANM: Adjacent normal mucosa; circRNA: Circular RNA; EMT: Epithelial-mesenchymal transition; qPCR: Quantitative real-time PCR; FISH: Fluorescence in situ hybridization; RIP: RNA immunoprecipitation; 3' UTR: 3'-untranslated region; mTOR: Mammalian target of rapamycin; DAPI: 4',6-diamidino-2-phenylindole

\section{Acknowledgments}

We thank Prof. Tao Bai from the Department of Pathology, First Hospital of Shanxi Medical University for pathological analysis of LSCC sections.

\section{Authors' contributions}

YYW conceived the study and participated in the study design. WG, HNG $M N, X W Z, Y L Z, X T X, L H, Y Z$ and LL performed cell culture, colony formation qPCR, western blots, RIP, luciferase reporter and RNA pulldown assays. XWZ, YFB, XYG and ZXL performed bioinformatics analysis, IHC staining and pathological diagnosis. WG, HNG, MN, LH and YZ performed high-content screening and autophagic flux analysis. WG and HNG contributed to FISH experiments. WG and HNG performed xenograft experiments. YFB, XYG, XWZ and LL contributed to the clinical samples collection, follow-up and clinical data analysis. YYW, HNG, YLZ and JMC performed primer design and plasmid construction. WG, YJG, JMC and YYW analyzed the data, organized figures and wrote the manuscript. All authors read and approved the final manuscript.

\section{Funding}

This work was supported by the National Natural Science Foundation of China (No. 81602394), Shanxi Province Science Foundation for Excellent Young Scholars (No. 201901D211486), The Excellent talent science and technology innovation project of Shanxi Province (No. 201605D211029, 201705D211018), Shanxi Province Science Foundation for Youths (No.201901D211490), Shanxi Province Scientific and Technological Achievements Transformation Guidance Foundation (No. 201804D131043), Youth Top Talent Program Fund of Shanxi Province (No. 2018), Fund of Shanxi "1331" Project (No. 2018), Research Project Supported by Shanxi Scholarship Council of China (No. 2020165).

\section{Availability of data and materials}

RNA sequencing raw data and normalized results were deposited at GEO database (GSE132222, GSE130605, GSE127165, and GSE133632). All data that support the findings of this study are available from the corresponding authors upon reasonable request.

\section{Ethics approval and consent to participate}

The clinical samples were collected from patients after informed consent was obtained. Studies were performed with the approval of the medical ethics committee of First Hospital of Shanxi Medical University. Animal experiments were conducted according to the Health Guide for the Care and Use of Laboratory Animals approved by the Animal Experimental Research Ethics Committee of Shanxi Medical University.

\section{Consent for publication}

Not applicable.

\section{Competing interests}

The authors declare that they have no competing interests.

\section{Author details}

${ }^{1}$ Shanxi Key Laboratory of Otorhinolaryngology Head and Neck Cancer, First Hospital of Shanxi Medical University, Taiyuan 030001, China. ${ }^{2}$ Shanxi Province Clinical Medical Research Center for Precision Medicine of Head and Neck Cancer, First Hospital of Shanxi Medical University, Taiyuan 030001, China. ${ }^{3}$ Department of Otolaryngology Head \& Neck Surgery, First Hospital of Shanxi Medical University, Taiyuan 030001, China. ${ }^{4}$ Key Laboratory of Cellular Physiology, Ministry of Education, Shanxi Medical University, Taiyuan 030001, China. ${ }^{5}$ Department of Cell Biology and Genetics, Basic Medical School of Shanxi Medical University, Taiyuan 030001, China. ${ }^{6}$ Department of Pathology, Shanxi Cancer Hospital, Shanxi Medical University, Taiyuan 030013, China

${ }^{7}$ Department of Physiology, Shanxi Medical University, Taiyuan 030001, China. ${ }^{8}$ Department of Biochemistry \& Molecular Biology, Shanxi Medical University, Taiyuan 030001, China.

Received: 17 August 2020 Accepted: 3 November 2020

Published online: 24 November 2020

\section{References}

1. Steuer CE, El-Deiry M, Parks JR, Higgins KA, Saba NF. An update on larynx cancer. CA Cancer J Clin. 2017:67:31-50

2. Yuan Z, Xiu C, Song K, Pei R, Miao S, Mao X, Sun J, Jia S. Long non-coding RNA AFAP1-AS1/miR-320a/RBPJ axis regulates laryngeal carcinoma cell stemness and chemoresistance. J Cell Mol Med. 2018;22:4253-62. 
3. Gao W, Zhang Y, Niu M, Bo Y, Li H, Xue X, Lu Y, Zheng X, Tang Y, Cui J, et al. Identification of miR-145-5p-centered competing endogenous RNA network in laryngeal squamous cell carcinoma. Proteomics. 2019;19: e1900020.

4. Antonioli M, Di Rienzo M, Piacentini M, Fimia GM. Emerging mechanisms in initiating and terminating autophagy. Trends Biochem Sci. 2017:42:28-41.

5. Levy J, Towers CG, Thorburn A. Targeting autophagy in cancer. Nat Rev Cancer. 2017;17:528-42.

6. Patop IL, Wüst S, Kadener S. Past, present, and future of circRNAs. EMBO J. 2019;38:e100836

7. Kristensen LS, Andersen MS, Stagsted L, Ebbesen KK, Hansen TB, Kjems J. The biogenesis, biology and characterization of circular RNAs. Nat Rev Genet. 2019;20:675-91

8. Zhu KP, Zhang CL, Ma XL, Hu JP, Cai T, Zhang L. Analyzing the interactions of mRNAs and ncRNAs to predict competing endogenous RNA networks in osteosarcoma chemo-resistance. Mol Ther. 2019;27:518-30.

9. Huang $\mathrm{A}$, Zheng $\mathrm{H}, \mathrm{Wu} Z$, Chen $\mathrm{M}$, Huang Y. Circular RNA-protein interactions: functions, mechanisms, and identification. Theranostics. 2020;10:3503-17.

10. Pan Z, Cai J, Lin J, Zhou H, Peng J, Liang J, Xia L, Yin Q, Zou B, Zheng J, et al. A novel protein encoded by circFNDC3B inhibits tumor progression and EMT through regulating snail in colon cancer. Mol Cancer. 2020;19:71.

11. Du WW, Yang W, Li X, Awan FM, Yang Z, Fang L, Lyu J, Li F, Peng C, Krylov $\mathrm{SN}$, et al. A circular RNA circ-DNMT1 enhances breast cancer progression by activating autophagy. Oncogene. 2018;37:5829-42.

12. Zhou ZB, Niu YL, Huang GX, Lu JJ, Chen A, Zhu L. Silencing of circRNA.2837 plays a protective role in sciatic nerve injury by sponging the miR-34 family via regulating neuronal autophagy. Mol Ther Nucleic Acids. 2018;12:718-29.

13. Han B, Zhang Y, Zhang Y, Bai Y, Chen X, Huang R, Wu F, Leng S, Chao J,

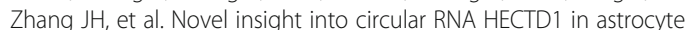
activation via autophagy by targeting MIR142-TIPARP: implications for cerebral ischemic stroke. Autophagy. 2018;14:1164-84.

14. Wu CP, Zhou L, Gong HL, Du HD, Tian J, Sun S, Li JY. Establishment and characterization of a novel HPV-negative laryngeal squamous cell carcinoma cell line, FD-LSC-1, with missense and nonsense mutations of TP53 in the DNA-binding domain. Cancer Lett. 2014;342:92-103.

15. Chu KJ, Ma YS, Jiang XH, Wu TM, Wu ZJ, Li ZZ, Wang JH, Gao QX, Yi B, Shi $Y$, et al. Whole-Transcriptome sequencing identifies key differentially expressed mRNAs, miRNAs, IncRNAs, and circRNAs associated with CHOL. Mol Ther Nucleic Acids. 2020;21:592-603.

16. Guan B, Wang TL, leM S. ARID1A, a factor that promotes formation of SWI/ SNF-mediated chromatin remodeling, is a tumor suppressor in gynecologic cancers. Cancer Res. 2011;71:6718-27.

17. Gan X, Zhu H, Jiang X, Obiegbusi SC, Yong M, Long X, Hu J. CircMUC16 promotes autophagy of epithelial ovarian cancer via interaction with ATG13 and miR-199a. Mol Cancer. 2020;19:45.

18. Subramanian A, Tamayo P, Mootha VK, Mukherjee S, Ebert BL, Gillette MA Paulovich A, Pomeroy SL, Golub TR, Lander ES, et al. Gene set enrichment analysis: a knowledge-based approach for interpreting genome-wide expression profiles. Proc Natl Acad Sci U S A. 2005;102:15545-50.

19. Yu G, Wang LG, Han Y, He QY. clusterProfiler: an R package for comparing biological themes among gene clusters. OMICS. 2012;16:284-7.

20. Bandyopadhyay S, Mitra R. TargetMiner: microRNA target prediction with systematic identification of tissue-specific negative examples. Bioinformatics. 2009;25:2625-31.

21. Dudekula DB, Panda AC, Grammatikakis I, De S, Abdelmohsen K, Gorospe M. Circlnteractome: a web tool for exploring circular RNAs and their interacting proteins and microRNAs. RNA Biol. 2016:13:34-42.

22. Ichimura Y, Kominami E, Tanaka K, Komatsu M. Selective turnover of p62/ A170/SQSTM1 by autophagy. Autophagy. 2008;4:1063-6.

23. Siadous FA, Cantet F, Van Schaik E, Burette M, Allombert J, Lakhani A, Bonaventure B, Goujon C, Samuel J, Bonazzi M, et al. Coxiella effector protein CvpF subverts RAB26-dependent autophagy to promote vacuole biogenesis and virulence. Autosphagy. 2020:1-17. https://doi.org/10.1080/ 15548627.2020 .1728098$.

24. Li Y, Chen B, Huang S. Identification of circRNAs for miRNA targets by Argonaute2 RNA Immunoprecipitation and luciferase screening assays. Methods Mol Biol. 1724;2018:209-18.

25. Gao W, Zhang C, Li W, Li H, Sang J, Zhao Q, Bo Y, Luo H, Zheng X, Lu Y, et al. Promoter methylation-regulated miR-145-5p inhibits laryngeal squamous cell carcinoma progression by targeting FSCN1. Mol Ther. 2019; 27:365-79.
26. Qu L, Li G, Xia D, Hongdu B, Xu C, Lin X, Chen Y. PRKCI negatively regulates autophagy via PIK3CAVAKT-MTOR signaling. Biochem Biophys Res Commun. 2016;470:306-12.

27. Zhou J, Zhou L, Zhang D, Tang WJ, Tang D, Shi XL, Yang Y, Zhou L, Liu F, Yu Y, et al. BCL11A promotes the progression of laryngeal squamous cell carcinoma. Front Oncol. 2020;10:375.

28. Song P, Li Y, Dong Y, Liang Y, Qu H, Qi D, Lu Y, Jin X, Guo Y, Jia Y, et al. Estrogen receptor $\beta$ inhibits breast cancer cells migration and invasion through CLDN6-mediated autophagy. J Exp Clin Cancer Res. 2019;38:354.

29. Bell ES, Coelho PP, Ratcliffe C, Rajadurai CV, Peschard P, Vaillancourt R, Zuo D, Park M. LC3C-Mediated Autophagy Selectively Regulates the Met RTK and HGF-Stimulated Migration and Invasion. Cell Rep. 2019;29:4053-68.e6.

30. Levy JM, Thompson JC, Griesinger AM, Amani V, Donson AM, Birks DK Morgan MJ, Mirsky DM, Handler MH, Foreman NK, et al. Autophagy inhibition improves chemosensitivity in BRAF(V600E) brain tumors. Cancer Discov. 2014;4:773-80

31. Wang D, Zhang P, Xu X, Wang J, Wang D, Peng P, Zheng C, Meng QJ, Yang L, Luo Z. Knockdown of cytokeratin 8 overcomes chemoresistance of chordoma cells by aggravating endoplasmic reticulum stress through PERK elF2a arm of unfolded protein response and blocking autophagy. Cell Death Dis. 2019;10:887

32. Li H, Chen L, Li JJ, Zhou Q, Huang A, Liu WW, Wang K, Gao L, Qi ST, Lu YT. miR-519a enhances chemosensitivity and promotes autophagy in glioblastoma by targeting STAT3/BCl2 signaling pathway. J Hematol Oncol. 2018;11:70.

33. Zhu JF, Huang W, Yi HM, Xiao T, Li JY, Feng J, Yi H, Lu SS, Li XH, Lu RH, et al. Annexin A1-suppressed autophagy promotes nasopharyngeal carcinoma cell invasion and metastasis by PI3KAKT signaling activation. Cell Death Dis. 2018;9:1154

34. Wang J, Zhao X, Wang Y, Ren F, Sun D, Yan Y, Kong X, Bu J, Liu M, Xu S. circRNA-002178 act as a ceRNA to promote PDL1/PD1 expression in lung adenocarcinoma. Cell Death Dis. 2020;11:32.

35. Ou R, Lv J, Zhang Q, Lin F, Zhu L, Huang F, Li X, Li T, Zhao L, Ren Y, et al. circAMOTL1 motivates AMOTL1 expression to facilitate cervical Cancer growth. Mol Ther Nucleic Acids. 2020;19:50-60.

36. Liang G, Ling Y, Mehrpour M, Saw PE, Liu Z, Tan W, Tian Z, Zhong W, Lin W, Luo Q, et al. Autophagy-associated circRNA circCDYL augments autophagy and promotes breast cancer progression. Mol Cancer. 2020;19:65.

37. Higashi K, Yamada Y, Minatoguchi S, Baba S, Iwasa M, Kanamori H, Kawasaki M, Nishigaki K, Takemura G, Kumazaki M, et al. MicroRNA-145 repairs infarcted myocardium by accelerating cardiomyocyte autophagy. Am J Physiol Heart Circ Physiol. 2015;309:H1813-26.

38. Xiang J, Jiang T, Zhang W, Xie W, Tang X, Zhang J. Human umbilical cordderived mesenchymal stem cells enhanced HK-2 cell autophagy through MicroRNA-145 by inhibiting the PI3K/AKT/mTOR signaling pathway. Exp Cell Res. 2019:378:198-205.

39. Yin N, Liu Y, Khoor A, Wang $X$, Thompson EA, Leitges M, Justilien V, Weems C, Murray NR, Fields AP. Protein Kinase CI and Wnt/ $\beta$-Catenin Signaling: Alternative Pathways to Kras/Trp53-Driven Lung Adenocarcinoma. Cancer Cell. 2019;36:156-67.e7.

40. Sarkar S, Bristow CA, Dey P, Rai K, Perets R, Ramirez-Cardenas A, Malasi S, Huang-Hobbs E, Haemmerle M, Wu SY, et al. PRKCI promotes immune suppression in ovarian cancer. Genes Dev. 2017;31:1109-21.

41. Kikuchi K, Soundararajan A, Zarzabal LA, Weems CR, Nelon LD, Hampton ST, Michalek JE, Rubin BP, Fields AP, Keller C. Protein kinase C iota as a therapeutic target in alveolar rhabdomyosarcoma. Oncogene. 2013;32:286-95.

\section{Publisher's Note}

Springer Nature remains neutral with regard to jurisdictional claims in published maps and institutional affiliations. 